\title{
LOCAL AND NONLOCAL WEIGHTED $p$-LAPLACIAN EVOLUTION EQUATIONS WITH NEUMANN BOUNDARY CONDITIONS
}

\author{
F. ANDREU, J. M. MAZÓN, J. D. ROSSI AND J. TOLEDO
}

Abstract. In this paper we study existence and uniqueness for solutions of the nonlocal diffusion equation with Neumann boundary conditions

$$
\left.u_{t}(t, x)=\int_{\Omega} J(x-y) g \quad \frac{x+y}{2}|u(t, y)-u(t, x)|^{p-2}(u(t, y)-u(t, x)) d y \quad \text { in }\right] 0, T[\times \Omega,
$$

and for solutions of its local counterpart

$$
\begin{cases}u_{t}=\operatorname{div} g|\nabla u|^{p-2} \nabla u & \text { in }] 0, T[\times \Omega, \\ g|\nabla u|^{p-2} \nabla u \cdot \eta=0 & \text { on }] 0, T[\times \partial \Omega .\end{cases}
$$

We consider $1 \leq p<\infty$ and $g \geq 0$. We pay special attention to the case in which $g$ vanishes somewhere in $\Omega$, even in a set of positive measure.

\section{INTRODUCTION}

In this paper we have two main goals. As a first goal, we study the following nonlocal nonlinear diffusion problem with homogeneous Neumann boundary condition

$$
P_{p}^{J, g}\left(u_{0}\right) \quad\left\{\begin{array}{l}
u_{t}(t, x)=\int_{\Omega} J(x-y) g\left(\frac{x+y}{2}\right)|u(t, y)-u(t, x)|^{p-2}(u(t, y)-u(t, x)) d y, \\
u(x, 0)=u_{0}(x),
\end{array}\right.
$$

where $g \in L^{\infty}\left(\mathbb{R}^{N}\right), g \geq 0$ a.e. in $\mathbb{R}^{N}, 1 \leq p<+\infty, \Omega \subset \mathbb{R}^{N}$ is a bounded smooth domain and the kernel $J$ satisfies

(HJ) $J: \mathbb{R}^{N} \rightarrow \mathbb{R}$ is a nonnegative continuous radial function with compact support, $J(0)>0$ and $\int_{\mathbb{R}^{N}} J(z) d z=1$.

As a second goal, we also study the local counterpart, that is, the following local diffusion equation with homogeneous Neumann boundary condition

$$
N_{p}^{g}\left(u_{0}\right) \quad \begin{cases}u_{t}=\operatorname{div}\left(g|\nabla u|^{p-2} \nabla u\right) & \text { in }] 0, T[\times \Omega, \\ g|\nabla u|^{p-2} \nabla u \cdot \eta=0 & \text { on }] 0, T[\times \partial \Omega, \\ u(x, 0)=u_{0}(x) & \text { in } \Omega\end{cases}
$$

where $\eta$ is the unit outward normal on $\partial \Omega$.

As we will show here, these two problems are related in the following way: solutions of the nonlocal problem converge to solutions of the local one when the kernel $J$ is rescaled in a suitable way.

Key words and phrases. Nonlocal diffusion, $p$-Laplacian, Total variation flow, Neumann boundary conditions. 2000 Mathematics Subject Classification. 45G10, 45J05, 47H06. 
In these two problems we deal with a non-homogeneous diffusion coefficient, given by the function $g$, that we assume to be bounded and nonnegative, but we include here the case in which $g$ vanishes in a subset of $\Omega$ that can even have positive measure. In this case we face new technical difficulties since we lost the coercivity of the associated functional in the usual Sobolev or Lebesgue spaces. These difficulties are overcome using weighted Sobolev or Lebesgue spaces with appropriate hypothesis on $g$ that involve weights in Muckenhoupt's $A_{p}$ classes.

Observe that for homogeneous diffusion, $g=1$, the operator in the local problem is given by $\operatorname{div}\left(g|\nabla u|^{p-2} \nabla u\right)=\operatorname{div}\left(|\nabla u|^{p-2} \nabla u\right)=\Delta_{p} u$, that is, the well-known $p$-Laplacian of $u$. Also note that when $p=2$ both problems become linear. In the case $g=1$ the study of such problems has been done in [7] for the nonlocal problem while the local problem is a well known classical problem (see for instance, [47], [48]). Moreover, in this case, it is proved in [7] that under an appropriate rescaling of the kernel $J$, the solutions of the rescaled nonlocal problems, when the scale parameter (that measure the size of the support of $J$ ) tends to zero, converge to the solutions of the local problem.

One of the main results of this paper is to prove a similar convergence result for $P_{p}^{J, g}\left(u_{0}\right)$, where $g$ can vanish in a subset of $\Omega$ of positive measure. This fact turns the whole issue more involved since the nonlocal problem, in contrast with what happens in general for the local one, takes into account the part of the domain where the diffusion coefficient $g$ is null, that is, this part of the domain plays a role in the nonlocal diffusion case.

The case $p=1$ is somehow different from the case $p>1$. In fact, for $p=1$ we need to work in weighted $B V$ spaces (that is, weighted bounded variation spaces), an issue that forces us to introduce some delicate results from measure theory. The local problem for $g=1$ with $p=1$, that is, the Neumann problem for the total variation flow, was studied in [3] (see also [4]), motivated by problems in image processing. This PDE appears when one uses the steepest descent method to minimize the total variation, a method introduced by L. Rudin, S. Osher and E. Fatemi [43] in the context of image denoising and reconstruction. The use of weighted total variational functionals in image processing began with the seminal work of V. Caselles, R. Kimmel and G. Sapiro ([25], [26]) on geodesic active contours. Also in the the unpublished paper [46] the weighted total variational functionals in image processing was considered (see also [27]). Until the recent paper of V. Caselles, G. Facciolo and E. Meinhardt [24], it was always supposed that the weight $g$ is positive. In [24] it is admitted that $g$ can be null in a set of positive measure. Here also this possibility is considered.

To finish this introduction, let us briefly introduce some references for the prototype of nonlocal problem that will be considered along this work. Nonlocal evolution equations of the form $u_{t}(t, x)=(J * u-u)(t, x)=\int_{\mathbb{R}^{N}} J(x-y) u(t, y) d y-u(t, x)$, and variations of it, have been recently widely used to model diffusion processes. More precisely, as stated in [39], if $u(t, x)$ is thought of as a density at the point $x$ at time $t$ and $J(x-y)$ is thought of as the probability distribution of jumping from location $y$ to location $x$, then $\int_{\mathbb{R}^{N}} J(y-x) u(t, y) d y=(J * u)(t, x)$ is the rate at which individuals are arriving at position $x$ from all other places and $-u(t, x)=-\int_{\mathbb{R}^{N}} J(y-x) u(t, x) d y$ is the rate at which they are leaving location $x$ to travel to all other sites. This consideration, in the absence of external or internal sources, leads immediately to the fact that the density $u$ satisfies the equation $u_{t}=J * u-u$. Nonlocal diffusion equations have been recently widely studied and have connections with probability theory (for example, Levy processes are related to the fractional Laplacian), see, [6], [7], [8], [12], [13], [21], [22], [23], [28], [29], [32], [33], [34], [39], [44], [45] and references therein. 
Especially related to the nonlocal problem $P_{p}^{J, g}\left(u_{0}\right)$ are references [7], [8] and [9], in fact, this work can be viewed as a natural continuation of those papers. In [7], from where we borrow some ideas, the nonlocal problem with $g=1$ is studied. In [8] we deal with the limit as $p \rightarrow \infty$ of solutions to nonlocal problems and in [9] Dirichlet boundary conditions are imposed. All these previous works deal with $g=1$. Concerning inhomogeneous nonlocal diffusion we quote [31] and [35] where the authors study the nonlocal analogous to the linear equation $u_{t}=\Delta\left(g^{2} u\right)$ in the whole $\mathbb{R}^{N}$.

Organization of the paper. The rest of the paper is organized as follows: in Section 2 we prove existence and uniqueness for the nonlocal problem with $p>1$. Section 3 deal with the local problem for $p>1$ and in Section 4 we show the convergence of the nonlocal problems to the local problem for $p>1$. In sections 5,6 and 7 we deal with analogous questions for $p=1$. We prefer to present the results for $p=1$ in separate sections since, as we have mentioned, in this case the use of $B V$ functions introduces technical differences that we want to highlight.

\section{Existence AND Uniqueness of SOlUtions FOR THE NONLOCAL PROBlEM. \\ THE CASE $p>1$}

Let us begin this section by collecting some preliminaries and notations that will be used in the sequel. We denote by $J_{0}$ and $P_{0}$ the following sets of functions,

$$
\begin{gathered}
J_{0}=\{j: \mathbb{R} \rightarrow[0,+\infty], \text { convex and lower semi-continuos with } j(0)=0\}, \\
P_{0}=\left\{q \in C^{\infty}(\mathbb{R}): 0 \leq q^{\prime} \leq 1, \operatorname{supp}\left(q^{\prime}\right) \text { is compact, and } 0 \notin \operatorname{supp}(q)\right\} .
\end{gathered}
$$

In [15] the following relation for $u, v \in L^{1}(\Omega)$ is defined,

$$
u \ll v \text { if and only if } \int_{\Omega} j(u) d x \leq \int_{\Omega} j(v) d x \quad \text { for all } j \in J_{0},
$$

and the following facts are proved.

Proposition 2.1. Let $\Omega$ be a bounded domain in $\mathbb{R}^{N}$.

(i) For any $u, v \in L^{1}(\Omega)$, if $\int_{\Omega} u q(u) \leq \int_{\Omega} v q(u)$ for all $q \in P_{0}$, then $u \ll v$.

(ii) If $u, v \in L^{1}(\Omega)$ and $u \ll v$, then $\|u\|_{r} \leq\|v\|_{r}$ for any $r \in[1,+\infty]$.

(iii) If $v \in L^{1}(\Omega)$, then $\left\{u \in L^{1}(\Omega): u \ll v\right\}$ is a weakly compact subset of $L^{1}(\Omega)$.

In this section we deal with existence and uniqueness for solutions of the nonlocal problem $P_{p}^{J, g}\left(u_{0}\right)$ that will be understood according to the following definition.

Definition 2.2. Let $p>1$. A solution of the problem $P_{p}^{J, g}\left(u_{0}\right)$ in $[0, T]$ is a function $u \in$ $W^{1,1}\left(0, T ; L^{1}(\Omega)\right)$ which satisfies $u(0, x)=u_{0}(x)$ a.e. $x \in \Omega$ and

$$
\left.u_{t}(t, x)=\int_{\Omega} J(x-y) g\left(\frac{x+y}{2}\right)|u(t, y)-u(t, x)|^{p-2}(u(t, y)-u(t, x)) d y \quad \text { a.e. in } \quad\right] 0, T[\times \Omega \text {. }
$$

To study the problem $P_{p}^{J, g}\left(u_{0}\right)$ we use Nonlinear Semigroup Theory. To this end we introduce in $L^{1}(\Omega)$ the following operator associated with our problem. 
Definition 2.3. Let $J$ satisfies (HJ), $g \in L^{\infty}\left(\mathbb{R}^{N}\right), g \geq 0$ a.e., and $1<p<+\infty$. We define in $L^{1}(\Omega)$ the operator $B_{p}^{J, g}$ by

$$
B_{p}^{J, g} u(x)=-\int_{\Omega} J(x-y) g\left(\frac{x+y}{2}\right)|u(y)-u(x)|^{p-2}(u(y)-u(x)) d y, \quad x \in \Omega .
$$

Remark 2.4. It is easy to see that,

1. $B_{p}^{J, g}$ is positively homogeneous of degree $p-1$,

2. $L^{p-1}(\Omega) \subset \operatorname{Dom}\left(B_{p}^{J, g}\right)$, if $p>2$,

3. for $1<p \leq 2, \operatorname{Dom}\left(B_{p}^{J, g}\right)=L^{1}(\Omega)$ and $B_{p}^{J, g}$ is closed in $L^{1}(\Omega) \times L^{1}(\Omega)$.

We have the following monotonicity lemma, whose proof is straightforward.

Lemma 2.5. Let $T: \mathbb{R} \rightarrow \mathbb{R}$ a nondecreasing function. Then,

(i) for every $u, v \in L^{p}(\Omega)$ such that $T(u-v) \in L^{p}(\Omega)$, it holds

$$
\begin{aligned}
& \int_{\Omega}(\left.B_{p}^{J, g} u(x)-B_{p}^{J, g} v(x)\right) T(u(x)-v(x)) d x= \\
& \frac{1}{2} \int_{\Omega} \int_{\Omega} J(x-y) g\left(\frac{x+y}{2}\right)(T(u(y)-v(y))-T(u(x)-v(x))) \times \\
& \quad \times\left(|u(y)-u(x)|^{p-2}(u(y)-u(x))-|v(y)-v(x)|^{p-2}(v(y)-v(x))\right) d y d x .
\end{aligned}
$$

(ii) Moreover, if $T$ is bounded, (2.1) holds for $u, v \in \operatorname{Dom}\left(B_{p}^{J, g}\right)$.

Following ideas from [7] we have that $B_{p}^{J, g}$ is completely accretive and verifies the range condition $L^{p}(\Omega) \subset \operatorname{Ran}\left(I+B_{p}^{J, g}\right)$. In short, this means that for any $\phi \in L^{p}(\Omega)$ there is a unique solution of the problem $u+B_{p}^{J, g} u=\phi$ and the resolvent $\left(I+B_{p}^{J, g}\right)^{-1}$ is a contraction in $L^{q}(\Omega)$ for all $1 \leq q \leq+\infty$.

Theorem 2.6. The operator $B_{p}^{J, g}$ is completely accretive and verifies the range condition

$$
L^{p}(\Omega) \subset \operatorname{Ran}\left(I+B_{p}^{J, g}\right) .
$$

Proof. The proof mimics the one given for $g=1$ in [7]. We include it here for completeness. Given $u_{i} \in \operatorname{Dom}\left(B_{p}^{J, g}\right), i=1,2$ and $q \in P_{0}$, by the monotonicity Lemma 2.5, we have

$$
\int_{\Omega}\left(B_{p}^{J, g} u_{1}(x)-B_{p}^{J, g} u_{2}(x)\right) q\left(u_{1}(x)-u_{2}(x)\right) d x \geq 0
$$

from where it follows that $B_{p}^{J, g}$ is a completely accretive operator (see [15]).

To show that $B_{p}^{J, g}$ satisfies the range condition we have to prove that for any $\phi \in L^{p}(\Omega)$ there exists $u \in \operatorname{Dom}\left(B_{p}^{J, g}\right)$ such that $u=\left(I+B_{p}^{J, g}\right)^{-1} \phi$. Let us first take $\phi \in L^{\infty}(\Omega)$. Let $A_{n, m}: L^{p}(\Omega) \rightarrow L^{p^{\prime}}(\Omega)$ the continuous monotone operator defined by

$$
A_{n, m}(u):=T_{c}(u)+B_{p}^{J, g} u+\frac{1}{n}|u|^{p-2} u^{+}-\frac{1}{m}|u|^{p-2} u^{-},
$$

where $T_{c}(s)=\sup (-c, \inf (s, c))$. We have that $A_{n, m}$ is coercive in $L^{p}(\Omega)$. In fact,

$$
\lim _{\|u\|_{L^{p}(\Omega)} \rightarrow+\infty} \frac{\int_{\Omega} A_{n, m}(u) u}{\|u\|_{L^{p}(\Omega)}}=+\infty .
$$


Then, by Corollary 30 in [18], there exists $u_{n, m} \in L^{p}(\Omega)$, such that

$$
T_{c}\left(u_{n, m}\right)+B_{p}^{J, g} u_{n, m}+\frac{1}{n}\left|u_{n, m}\right|^{p-2} u_{n, m}^{+}-\frac{1}{m}\left|u_{n, m}\right|^{p-2} u_{n, m}^{-}=\phi .
$$

Using the monotonicity of $B_{p}^{J, g} u_{n, m}+\frac{1}{n}\left|u_{n, m}\right|^{p-2} u_{n, m}^{+}-\frac{1}{m}\left|u_{n, m}\right|^{p-2} u_{n, m}^{-}$, from Proposition 2.1, we obtain that $T_{c}\left(u_{n, m}\right) \ll \phi$ and therefore, taking $c>\|\phi\|_{L^{\infty}(\Omega)}, u_{n, m} \ll \phi$. Consequently,

$$
u_{n, m}+B_{p}^{J, g} u_{n, m}+\frac{1}{n}\left|u_{n, m}\right|^{p-2} u_{n, m}^{+}-\frac{1}{m}\left|u_{n, m}\right|^{p-2} u_{n, m}^{-}=\phi .
$$

Moreover, $u_{n, m}$ is increasing in $n$ and decreasing in $m$. As $u_{n, m} \ll \phi$, we can pass to the limit as $n \rightarrow \infty$ (using the monotone convergence to handle the term $B_{p}^{J, g} u_{n, m}$ ) obtaining a limit, $u_{m}$, that is a solution to

$$
u_{m}+B_{p}^{J, g} u_{m}-\frac{1}{m}\left|u_{m}\right|^{p-2} u_{m}^{-}=\phi .
$$

Using that $u_{m}$ is decreasing in $m$ we can pass again to the limit to obtain

$$
u+B_{p}^{J, g} u=\phi .
$$

Now, let us consider the general case $\phi \in L^{p}(\Omega)$. Take $\phi_{n} \in L^{\infty}(\Omega), \phi_{n} \rightarrow \phi$ in $L^{p}(\Omega)$. Then, by our previous step, there exists $u_{n}=\left(I+B_{p}^{J, g}\right)^{-1} \phi_{n}, u_{n} \ll \phi_{n}$. Since $B_{p}^{J, g}$ is completely accretive, $u_{n} \rightarrow u$ in $L^{p}(\Omega)$, also $B_{p}^{J, g} u_{n} \rightarrow B_{p}^{J, g} u$ in $L^{p^{\prime}}(\Omega)$ and we conclude that $u+B_{p}^{J, g} u=\phi$, $u \ll \phi$.

As a consequence of this result we get the following existence and uniqueness theorem for the evolution problem.

Theorem 2.7. Assume $p>1$. Let $T>0$ and $u_{0} \in L^{1}(\Omega)$. Then, there exists a unique mild solution $u$ of

$$
\left\{\begin{array}{l}
\left.u^{\prime}(t)+B_{p}^{J, g} u(t)=0, \quad t \in\right] 0, T[ \\
u(0)=u_{0}
\end{array}\right.
$$

Moreover,

(1) if $u_{0} \in L^{p}(\Omega)$, the unique mild solution $u$ of (2.3) is a solution of $P_{p}^{J, g}\left(u_{0}\right)$ in the sense of Definition 2.2. If $1<p \leq 2$, this is true for any $u_{0} \in L^{1}(\Omega)$.

(2) Let $u_{i_{0}} \in L^{1}(\Omega), i=1,2$, and $u_{i}$ a solution in $[0, T]$ of $P_{p}^{J, g}\left(u_{i_{0}}\right), i=1,2$. Then

$$
\left.\int_{\Omega}\left(u_{1}(t)-u_{2}(t)\right)^{+} \leq \int_{\Omega}\left(u_{10}-u_{20}\right)^{+} \quad \text { for every } t \in\right] 0, T[.
$$

Moreover, for $q \in[1,+\infty]$, if $u_{i 0} \in L^{q}(\Omega), i=1,2$, then

$$
\left.\left\|u_{1}(t)-u_{2}(t)\right\|_{L^{q}(\Omega)} \leq\left\|u_{10}-u_{20}\right\|_{L^{q}(\Omega)} \quad \text { for every } t \in\right] 0, T[.
$$

Proof. As a consequence of Theorem 2.6 we get the existence of mild solution of (2.3) (see [16] and [15]). On the other hand, $u(t)$ is a solution of $P_{p}^{J, g}\left(u_{0}\right)$ if and only if $u(t)$ is a strong solution of the abstract Cauchy problem (2.3). Now, due to the complete accretivity of $B_{p}^{J, g}$ and the range condition $(2.2), u(t)$ is a strong solution (see [15]). Moreover, in the case $1<p \leq 2$, since $\operatorname{Dom}\left(B_{p}^{J, g}\right)=L^{1}(\Omega)$ and $B_{p}^{J, g}$ is closed in $L^{1}(\Omega) \times L^{1}(\Omega)$, the result holds for $L^{1}$-data. Finally, the contraction principle is a consequence of the general Nonlinear Semigroup Theory. 


\section{The Local Problem for $p>1$}

We consider now the local evolution equation with homogeneous Neumann boundary conditions

$$
N_{p}^{g}\left(u_{0}\right) \quad \begin{cases}u_{t}=\operatorname{div}\left(g|D u|^{p-2} D u\right) & \text { in }] 0, T[\times \Omega, \\ g|D u|^{p-2} D u \cdot \eta=0 & \text { on }] 0, T[\times \partial \Omega \\ u(\cdot, 0)=u_{0} \in L^{1}(\Omega) & \text { in } \Omega\end{cases}
$$

where $\Omega$ is a bounded smooth domain, $\eta$ is the unit outward normal on $\partial \Omega$ and $g$ verifies

$$
\left[\begin{array}{l}
g \in L^{\infty}(\Omega), \\
g>0 \text { a.e. in } S, g=0 \text { a.e. in } \Omega \backslash S \text {, being } S \text { a smooth domain contained in } \Omega,
\end{array}\right.
$$

and

$$
g^{\frac{1}{1-p}} \in L^{1}(S) .
$$

We will work in the following weighted Sobolev space.

Definition 3.1. Set $W_{g, S}^{1, p}(\Omega)$ the space of functions $u \in L^{p}(\Omega)$ such that the distributional derivatives $\frac{\partial u}{\partial x_{i}}$ satisfy

$$
g^{1 / p} \frac{\partial u}{\partial x_{i}} \in L^{p}(S), \quad i=1,2, \ldots, N .
$$

This space $W_{g, S}^{1, p}(\Omega)$ endowed with the norm

$$
\|u\|_{W_{g, S}^{1, p}(\Omega)}:=\left(\int_{\Omega}|u(x)|^{p} d x+\int_{S}|D u(x)|^{p} g(x) d x\right)^{\frac{1}{p}}
$$

is a Banach space.

Let us recall that $w$ is a weight in the Muckenhoupt's $A_{p}$-class, or an $A_{p}$-weight, if $w$ is a nonnegative, locally (Lebesgue) integrable function in $\mathbb{R}^{N}$ such that

$$
\sup \left(\frac{1}{|B|} \int_{B} w(x) d x\right)\left(\frac{1}{|B|} \int_{B} w(x)^{\frac{1}{1-p}} d x\right)^{p-1}=c_{w, p}<\infty,
$$

where the supremum is taken over all ball $B$ in $\mathbb{R}^{N}$.

We also assume that

(3.3) there exists a weight function $g_{0}$ in the Muckenhoupt's $A_{p}$-class such that $g_{0}=g$ in $S$.

This hypothesis implies (3.2) since $S$ is bounded. Moreover, under this hypothesis, functions in $W_{g, S}^{1, p}(\Omega) \cap L^{\infty}(\Omega)$ can be approximated in the $\|\cdot\|_{W_{g, S}^{1, p}(\Omega)}$-norm by smooth functions (see [30], [37], [41], [42] and references therein for related topics). Indeed, we have the following result.

Lemma 3.2. For any $u \in W_{g, S}^{1, p}(\Omega) \cap L^{\infty}(\Omega)$ there exists $\varphi_{n} \in C^{\infty}(\Omega)$ such that $\varphi_{n} \rightarrow u$ in $W_{g, S}^{1, p}(\Omega)$. 
Proof. Given $u \in W_{g, S}^{1, p}(\Omega) \cap L^{\infty}(\Omega)$, by the results in [30], $u_{\left.\right|_{S}}$ can be extended to a function $\tilde{u} \in$ $W_{g_{0}, \mathbb{R}^{N}}^{1, p}\left(\mathbb{R}^{N}\right) \cap L^{\infty}\left(\mathbb{R}^{N}\right)$ with $\|\tilde{u}\|_{W_{g_{0}, \mathbb{R}^{N}}^{1, p}\left(\mathbb{R}^{N}\right)} \leq K\|u\|_{W_{g, S}^{1, p}(S)}$ and $\|\tilde{u}\|_{L^{\infty}\left(\mathbb{R}^{N}\right)} \leq\|u\|_{L^{\infty}(S)}$, where $K$ is independent of $u$. Now, by the results of [42], $\tilde{u}$ can be approximated in the $W_{g_{0}, \mathbb{R}^{N}}^{1, p}\left(\mathbb{R}^{N}\right)$ norm by $C^{\infty}$ functions $\tilde{\varphi}_{n}$ that are uniformly bounded in $L^{\infty}$. On the other hand, $u_{\left.\right|_{\Omega \backslash S}}$ can be approximated in the $L^{p}$-norm by smooth functions $\hat{\varphi}_{n}$ uniformly bounded in $L^{\infty}$. Therefore, we can find $\varphi_{n}$ such that

$$
\varphi_{n}= \begin{cases}\tilde{\varphi}_{n} & \text { in } S \\ \hat{\varphi}_{n} & \text { in } \Omega \backslash\left(S+B\left(0, \frac{1}{n}\right)\right),\end{cases}
$$

and in such a way that $\varphi_{n}$ is smooth and uniformly $L^{\infty}$-bounded. We conclude that $\varphi_{n} \rightarrow u$ in $W_{g, S}^{1, p}(\Omega)$.

We use the following concept of solution for problem $N_{p}^{g}\left(u_{0}\right)$.

Definition 3.3. A function $u \in W^{1,1}\left(0, T ; L^{1}(\Omega)\right)$ is an entropy solution of problem $N_{p}^{g}\left(u_{0}\right)$ in ] $0, T\left[\right.$ if $u(0)=u_{0}, T_{k}(u(t)) \in W_{g, S}^{1, p}(\Omega)$ for every $k>0$ and

$$
\int_{\Omega} u^{\prime}(t) T_{k}(u(t)-\phi) d x+\int_{S} g(x)|D u(t)|^{p-2} D u(t) \cdot D\left(T_{k}(u(t)-\phi)\right) d x \leq 0,
$$

for every $\phi \in W_{g, S}^{1, p}(\Omega) \cap L^{\infty}(\Omega)$ and all $k>0$. Here $T_{k}(r)$ is the classical truncature function $T_{k}(r)=\sup \{\inf \{r, k\},-k\}$.

To get the existence of entropy solutions of problem $N_{p}^{g}\left(u_{0}\right)$ we use again the Nonlinear Semigroups Theory, so we start with the study of the elliptic problem

$$
E_{p}^{g}(f) \begin{cases}u-\operatorname{div}\left(g|D u|^{p-2} D u\right)=f & \text { in } \Omega, \\ g|D u|^{p-2} D u \cdot \eta=0 & \text { on } \partial \Omega .\end{cases}
$$

Let us now introduce the following operator related to the local problem.

Definition 3.4. For $p>1$ and $g$ satisfying (3.1) and (3.3), we define the operator $B_{p}^{g}$ in $L^{1}(\Omega)$ by the following rule: $(u, \hat{u}) \in B_{p}^{g}$ if and only if $u \in W_{g, S}^{1, p}(\Omega) \cap L^{\infty}(\Omega), \hat{u} \in L^{1}(\Omega)$ and

$$
\int_{S} g(x)|D u|^{p-2} D u \cdot D v d x=\int_{\Omega} \hat{u}(x) v(x) d x \quad \forall v \in W_{g, S}^{1, p}(\Omega) \cap L^{\infty}(\Omega) .
$$

Proposition 3.5. Assume $g$ satisfies (3.1) and (3.3). Then the operator $B_{p}^{g}$ is completely accretive and satisfies the range condition $L^{\infty}(\Omega) \subset R\left(I+B_{p}^{g}\right)$.

Proof. Given $\left(u_{i}, v_{i}\right) \in B_{p}^{g}, i=1,2$, for any $q \in C^{\infty}(\mathbb{R}), 0 \leq q^{\prime} \leq 1, \operatorname{supp}\left(q^{\prime}\right)$ compact, $0 \notin$ $\operatorname{supp}(q)$, we have that

$$
\begin{aligned}
& \int_{\Omega}\left(v_{1}(x)-v_{2}(x)\right) q\left(u_{1}(x)-u_{2}(x)\right) d x= \\
& \int_{S} g(x) q^{\prime}\left(u_{1}-u_{2}\right)\left(\left|D u_{1}\right|^{p-2} D u_{1}-\left|D u_{2}\right|^{p-2} D u_{2}\right) \cdot D\left(u_{1}-u_{2}\right) d x \geq 0,
\end{aligned}
$$

from where it follows that $B_{p}^{g}$ is a completely accretive operator (see [15]). 
For every $n \in \mathbb{N}$, let $\mathbf{a}_{n}(x, \xi):=\left(g(x)+\frac{1}{n}\right)\|\xi\|^{p-2} \xi$. We define the operator $A_{n}$ in $L^{1}(\Omega)$ by the rule, $(u, \hat{u}) \in A_{n}$ if and only if $u \in W^{1, p}(\Omega) \cap L^{\infty}(\Omega), \hat{u} \in L^{1}(\Omega)$ and

$$
\int_{\Omega}\left\langle\mathbf{a}_{n}(x, D u), D(u-\phi)\right\rangle \leq \int_{\Omega} \hat{u}(u-\phi) \quad \text { for every } \phi \in W^{1, p}(\Omega) \cap L^{\infty}(\Omega) .
$$

Then, by the results in [5], we have $A_{n}$ is a completely accretive operator satisfying the range condition $L^{\infty}(\Omega) \subset R\left(I+A_{n}\right)$. Consequently, given $f \in L^{\infty}(\Omega)$ there exists a unique $u_{n} \in$ $W^{1, p}(\Omega) \cap L^{\infty}(\Omega)$ such that

$$
\int_{\Omega}\left\langle\mathbf{a}_{n}\left(x, D_{n}\right), D\left(u_{n}-\phi\right)\right\rangle \leq \int_{\Omega}\left(f-u_{n}\right)\left(u_{n}-\phi\right) \quad \text { for every } \phi \in W^{1, p}(\Omega) \cap L^{\infty}(\Omega),
$$

or equivalently,

$$
\int_{\Omega} g(x)\left|D u_{n}\right|^{p-2} D u_{n} \cdot D v+\frac{1}{n} \int_{\Omega}\left|D u_{n}\right|^{p-2} D u_{n} \cdot D v=\int_{\Omega}\left(f-u_{n}\right) v
$$

for every $v \in W^{1, p}(\Omega) \cap L^{\infty}(\Omega)$. Moreover,

$$
u_{n} \ll f \quad \text { for every } n \in \mathbb{N} \text {. }
$$

By (3.5) it follows that

$$
\left\|u_{n}\right\|_{q} \leq\|f\|_{q} \quad \text { for every } n \in \mathbb{N}, \text { and all } 1 \leq q \leq \infty .
$$

Taking $v=u_{n}$ as test function in (3.4), we get

$$
\int_{\Omega} g(x)\left|D u_{n}\right|^{p} d x+\frac{1}{n} \int_{\Omega}\left|D u_{n}\right|^{p} d x \leq \int_{\Omega}\left(f-u_{n}\right) u_{n} d x \quad \text { for every } n \in \mathbb{N} .
$$

From (3.6), taking a subsequence if necessary, we have there exists $u \in L^{\infty}(\Omega)$ such that

$$
u_{n} \rightarrow u \quad \text { weakly in } L^{p}(\Omega) .
$$

On the other hand, by (3.6) and (3.7), we get

$$
\int_{\Omega} g(x)\left|D u_{n}\right|^{p} d x+\frac{1}{n} \int_{\Omega}\left|D u_{n}\right|^{p} d x \leq M \quad \text { for every } n \in \mathbb{N} .
$$

By (3.9) and Hölder's inequality we have

$$
\begin{aligned}
\left.\left|\frac{1}{n} \int_{\Omega}\right| D u_{n}\right|^{p-2} D u_{n} \cdot D v \mid & \leq \frac{M^{\frac{1}{p^{\prime}}}}{n^{\frac{1}{p}}}\|D v\|_{p} \quad \forall n \in \mathbb{N}, \\
\left\|g^{\frac{1}{p}}\left|D u_{n}\right|\right\|_{L^{p}(\Omega)} & \leq M^{\frac{1}{p}} \quad \forall n \in \mathbb{N}
\end{aligned}
$$

and

$$
\left\|g^{\frac{1}{p^{\prime}}}\left|D u_{n}\right|^{p-2} D u_{n}\right\|_{L^{p^{\prime}}\left(\Omega, \mathbb{R}^{N}\right)} \leq M^{\frac{1}{p^{\prime}}} \quad \forall n \in \mathbb{N} .
$$

From (3.11), taking a subsequence if necessary, we have that

$$
g^{\frac{1}{p}} \frac{\partial u_{n}}{\partial x_{i}} \rightarrow w_{i} \quad \text { weakly in } L^{p}(\Omega), \quad i=1, \ldots, N .
$$


Given $\varphi \in \mathcal{D}(S)$, by (3.2), we have $g^{-\frac{1}{p}} \varphi \in L^{p^{\prime}}(S)$. Then, having in mind (3.13), we obtain

$$
\begin{aligned}
\left\langle\frac{\partial u}{\partial x_{i}}, \varphi\right\rangle= & -\int_{S} u \frac{\partial \varphi}{\partial x_{i}} d x=-\lim _{n \rightarrow \infty} \int_{S} u_{n} \frac{\partial \varphi}{\partial x_{i}} d x=\lim _{n \rightarrow \infty} \int_{S} \frac{\partial u_{n}}{\partial x_{i}} \varphi d x \\
& =\lim _{n \rightarrow \infty} \int_{S} g^{\frac{1}{p}} \frac{\partial u_{n}}{\partial x_{i}} g^{-\frac{1}{p}} \varphi d x=\int_{S} w_{i} g^{-\frac{1}{p}} \varphi d x .
\end{aligned}
$$

Consequently, we get

$$
\frac{\partial u}{\partial x_{i}}=w_{i} g^{-\frac{1}{p}} \quad \text { in } \mathcal{D}^{\prime}(S), \quad i=1, \ldots, N .
$$

Hence, since $w_{i} \in L^{p}(\Omega)$ and $g^{-\frac{1}{p}} \in L^{p^{\prime}}(S)$, we obtain that $\frac{\partial u}{\partial x_{i}} \in L^{1}(S)$, and $u \in W^{1,1}(S)$. Moreover, since $g \in L^{\infty}\left(\mathbb{R}^{N}\right)$,

$$
g^{\frac{1}{p}} \frac{\partial u}{\partial x_{i}}=w_{i} \in L^{p}(S), \quad i=1, \ldots, N .
$$

Therefore $u \in W_{g, S}^{1, p}(\Omega)$. Moreover, by (3.13) and (3.14), we have

$$
g^{\frac{1}{p}} D u_{n} \rightarrow g^{\frac{1}{p}} D u \quad \text { weakly in } L^{p}\left(S, \mathbb{R}^{N}\right) .
$$

By (3.12), taking a subsequence if necessary, there exists $\mathbf{z} \in L^{p^{\prime}}\left(\Omega, \mathbb{R}^{N}\right)$ such that

$$
g^{\frac{1}{p^{\prime}}}\left|D u_{n}\right|^{p-2} D u_{n} \rightarrow \mathbf{z} \quad \text { weakly in } L^{p^{\prime}}\left(\Omega, \mathbb{R}^{N}\right) .
$$

Given $v \in W^{1, p}(\Omega)$, taking limit in (3.4) and having in mind (3.8), (3.10) and (3.16), we obtain

$$
\int_{\Omega} g(x)^{\frac{1}{p}} \mathbf{z} \cdot D v d x=\int_{\Omega}(f-u) v d x .
$$

Setting $v=u_{n}$ in (3.17), using (3.8) and (3.15), and taking limit we get

$$
\int_{S} g(x)^{\frac{1}{p}} \mathbf{z} \cdot D u d x=\int_{\Omega}(f-u) u d x
$$

Let us see that

$$
g^{\frac{1}{p}} \mathbf{z}=g|D u|^{p-2} D u \quad \text { a.e. in } S .
$$

To do that we apply Minty-Browder's method. Note first that from (3.5) and (3.8), taking limit in (3.7) we get

$$
\limsup _{n \rightarrow+\infty} \int_{\Omega} g(x)\left|D u_{n}\right|^{p} d x \leq \int_{\Omega}(f(x)-u(x)) u(x) d x .
$$

On the other hand, by monotonicity, we have

$$
\int_{S} g(x)|\rho|^{p-2} \rho \cdot\left(D u_{n}-\rho\right) d x \leq \int_{S} g(x)\left|D u_{n}\right|^{p-2} D u_{n} \cdot\left(D u_{n}-\rho\right) d x
$$

for all $\rho$ such that $g^{\frac{1}{p}} \rho \in L^{p}\left(S, \mathbb{R}^{N}\right)$. Then, assuming that $g^{\frac{1}{p}} \rho \in L^{p}\left(S, \mathbb{R}^{N}\right)$, from (3.16), (3.15) and (3.20), taking limit in (3.21), we obtain that

$$
\int_{S} g(x)|\rho|^{p-2} \rho \cdot(D u-\rho) d x \leq \int_{\Omega}(f(x)-u(x)) u(x) d x-\int_{\Omega} g(x)^{\frac{1}{p}} \mathbf{Z} \cdot \rho d x .
$$


Hence, having in mind (3.18), we get

$$
\int_{S} g(x)|\rho|^{p-2} \rho \cdot(D u-\rho) d x \leq \int_{S} g(x)^{\frac{1}{p}} \mathbf{Z} \cdot D u d x-\int_{S} g(x)^{\frac{1}{p}} \mathbf{z} \cdot \rho d x .
$$

Then, taking in (3.23) $\rho=D u-\lambda \xi$, for $\lambda>0$ and $\xi \in L^{p}\left(S, \mathbb{R}^{N}\right)$, we obtain that

$$
\int_{S} g(x)|D u-\lambda \xi|^{p-2}(D u-\lambda \xi) \cdot \xi d x \leq \int_{S} g(x)^{\frac{1}{p}} \mathbf{z} \cdot \xi d x .
$$

From here, letting $\lambda \rightarrow 0$,

$$
\int_{S} g(x)|D u|^{p-2} D u \cdot \xi d x \leq \int_{S} g(x)^{\frac{1}{p}} \mathbf{Z} \cdot \xi d x \quad \forall \xi \in L^{p}\left(S, \mathbb{R}^{N}\right),
$$

from where (3.19) follows. Then, by (3.17) and (3.19), we obtain that

$$
\int_{S} g(x)|D u|^{p-2} D u \cdot D v d x=\int_{\Omega}(f-u) v d x \quad \forall v \in W^{1, p}(\Omega) .
$$

Now, using the fact that $g$ in $S$ is the restriction of a weight of Muckenhoupt's $A_{p}$-class, by Lemma 3.2, any $v \in W_{g, S}^{1, p}(\Omega) \cap L^{\infty}(\Omega)$ can be approximated by smooth functions and then, from (3.24), we obtain

$$
\int_{S} g(x)|D u|^{p-2} D u \cdot D v d x=\int_{\Omega}(f-u) v d x \quad \forall v \in W_{g, S}^{1, p}(\Omega) \cap L^{\infty}(\Omega) .
$$

Therefore, $(u, f-u) \in B_{p}^{g}$, and consequently, $f \in R\left(I+B_{p}^{g}\right)$.

Working as in [5] we can obtain the following characterization of the closure $\mathcal{B}_{p}^{g}$ of the operator $B_{p}^{g}$ in $L^{1}(\Omega) \times L^{1}(\Omega)$. Indeed, as we are considering a weight $g$ that is strictly positive in $S$ and the corresponding integrals that involve $g$ take place in $S$ we can follows the arguments of [5] with minor modifications.

Proposition 3.6. The closure of $B_{p}^{g}$ in $L^{1}(\Omega) \times L^{1}(\Omega)$ is given by $(u, v) \in \mathcal{B}_{p}^{g}$ if $u, v \in L^{1}(\Omega)$, $T_{k}(u) \in W_{g, S}^{1, p}(\Omega)$ and

$$
\int_{S} g(x)|D u|^{p-2} D u \cdot D\left(T_{k}(u-\phi)\right) d x \leq \int_{\Omega} v T_{k}(u-\phi) d x
$$

for every $\phi \in W_{g, S}^{1, p}(\Omega) \cap L^{\infty}(\Omega)$ and all $k>0$.

Theorem 3.7. For any $u_{0} \in L^{1}(\Omega)$ and any $T>0$, the problem $N_{p}^{g}\left(u_{0}\right)$ has a unique entropy solution in $] 0, T\left[\right.$. Moreover, an $L^{1}$-contraction principle holds for such solutions.

Proof. As a consequence of Proposition 3.5 the operator $\mathcal{B}_{p}^{g}$ is $m$-completely accretive in $L^{1}(\Omega)$. On the other hand, it is easy to see that ${\overline{D\left(B_{p}^{g}\right)}}^{L^{1}(\Omega)}=L^{1}(\Omega)$. Therefore, using the Nonlinear Semigroup Theory (see [36] and [16]), for any $u_{0} \in L^{1}(\Omega)$, the abstract Cauchy problem associated to $N_{p}^{g}\left(u_{0}\right)$ has a unique mild solution given by the exponential formula $v(t)=\mathrm{e}^{-t \mathcal{B}_{p}^{g}} u_{0}$. Moreover, as the operator is homogeneous of degree $p-1$, this solution is the unique strong solution of such abstract problem (see [15]). Now, by Proposition 3.6, the concept of strong solution and the concept of entropy solution of $N_{p}^{g}\left(u_{0}\right)$ coincide. The contraction principle follows by the Nonlinear Semigroup Theory. 
Observe that, in fact, a solution $u$ of $E_{p}^{g}(f)$ satisfies

$$
u=f \quad \text { a.e. in } \Omega \backslash S
$$

and $u_{\left.\right|_{S}}$ is a solution of

$$
\begin{cases}u-\operatorname{div}\left(g|D u|^{p-2} D u\right)=f & \text { in } S, \\ g|D u|^{p-2} D u \cdot \eta=0 & \text { on } \partial S .\end{cases}
$$

We can think that $g$ is a space-depending diffusion coefficient such that it has broken its diffusivity to 0 in some parts, so $u=f$ where there is no diffusivity. For the parabolic problem $u$ must be equal to the initial condition in places where $g$ vanishes. However, if $|\Omega \backslash S|>0$, when dealing with the nonlocal problem, it is not true that in general $u=f$ in $\Omega \backslash S$, even if $\operatorname{supp}(J)$ is very "small", there exists $\mathcal{O} \subset \Omega \backslash S$ with $|\mathcal{O}|>0$ where $u$ may differ from $f$. So, the part where $g=0$ plays a role in the nonlocal problem. Nevertheless, in the next section we will see that, under rescaling, solutions to the nonlocal problems converge to solutions to the local one.

\section{Convergence of the nonlocal problems to the local problem. The Case $p>1$}

Our main goal in this section is to show that the problem $N_{p}^{g}\left(u_{0}\right)$ can be approximated by suitable nonlocal Neumann problems of the form $P_{p}^{J, g}\left(u_{0}\right)$.

Let us now give the reescaling procedure. For given $p>1$ and $J$, we consider the rescaled kernels

$$
J_{p, \varepsilon}(x):=\frac{C_{J, p}}{\varepsilon^{p+N}} J\left(\frac{x}{\varepsilon}\right),
$$

where $C_{J, p}^{-1}:=\frac{1}{2} \int_{\mathbb{R}^{N}} J(z)\left|z_{N}\right|^{p} d z$ is a normalizing constant.

Associated to these kernels we solve $P_{p}^{J, g}\left(u_{0}\right)$ with $J_{p, \varepsilon}$ instead of $J$ with the same initial condition $u_{0}$ and we obtain a solution $u_{\varepsilon}(t, x)$. Our main concern in this section is to show that $u_{\varepsilon}$ converge to $u$ as $\varepsilon \rightarrow 0$, being $u$ a solution of $N_{p}^{g}\left(u_{0}\right)$.

First, let us perform a formal calculation in one space dimension just to convince the reader that the convergence result is correct. Let $g(x)$ and $u(x)$ be smooth functions and consider

$$
A_{\varepsilon}(u)=\frac{1}{\varepsilon^{p+1}} \int_{\mathbb{R}} J\left(\frac{x-y}{\varepsilon}\right) g\left(\frac{x+y}{2}\right)|u(y)-u(x)|^{p-2}(u(y)-u(x)) d y .
$$

Changing variables, $y=x-\varepsilon z$, we get

$$
A_{\varepsilon}(u)=\frac{1}{\varepsilon^{p}} \int_{\mathbb{R}} J(z) g\left(x-\frac{\varepsilon z}{2}\right)|u(x-\varepsilon z)-u(x)|^{p-2}(u(x-\varepsilon z)-u(x)) d z .
$$

Now, we expand in powers of $\varepsilon$ to obtain

$$
\begin{aligned}
& |u(x-\varepsilon z)-u(x)|^{p-2}=\varepsilon^{p-2}\left|u^{\prime}(x) z-\frac{u^{\prime \prime}(x)}{2} \varepsilon z^{2}+O\left(\varepsilon^{2}\right)\right|^{p-2} \\
& =\varepsilon^{p-2}\left|u^{\prime}(x)\right|^{p-2}|z|^{p-2}-\varepsilon^{p-1}(p-2)\left|u^{\prime}(x) z\right|^{p-4} u^{\prime}(x) z \frac{u^{\prime \prime}(x)}{2} z^{2}+O\left(\varepsilon^{p}\right),
\end{aligned}
$$

and

$$
u(x-\varepsilon z)-u(x)=-\varepsilon u^{\prime}(x) z+\frac{u^{\prime \prime}(x)}{2} \varepsilon^{2} z^{2}+O\left(\varepsilon^{3}\right)
$$


on the other hand, since $g$ is smooth,

$$
g\left(x-\frac{\varepsilon z}{2}\right)=g(x)-g^{\prime}(x) \frac{\varepsilon z}{2}+O\left(\varepsilon^{2}\right) .
$$

Hence, (4.1) becomes

$$
\begin{aligned}
A_{\varepsilon}(u)= & -\frac{1}{\varepsilon} \int_{\mathbb{R}} J(z)|z|^{p-2} z d z\left[g(x)\left|u^{\prime}(x)\right|^{p-2} u^{\prime}(x)\right] \\
& +\frac{1}{2} \int_{\mathbb{R}} J(z)|z|^{p} d z\left[g(x)\left((p-2)\left|u^{\prime}(x)\right|^{p-2} u^{\prime \prime}(x)+\left|u^{\prime}(x)\right|^{p-2} u^{\prime \prime}(x)\right)\right] \\
& +\frac{1}{2} \int_{\mathbb{R}} J(z)|z|^{p} d z\left[g^{\prime}(x)\left(\left|u^{\prime}(x)\right|^{p-2} u^{\prime}(x)\right)\right]+O(\varepsilon) .
\end{aligned}
$$

Using that $J$ is radially symmetric, the first integral vanishes and therefore,

$$
\lim _{\varepsilon \rightarrow 0} A_{\varepsilon}(u)=C\left(g(x)\left|u^{\prime}(x)\right|^{p-2} u^{\prime}(x)\right)^{\prime},
$$

where the constant $C$ is given by $C=\frac{1}{2} \int_{\mathbb{R}} J(z)|z|^{p} d z$.

To do this formal calculation rigorous we need to obtain the following result which is a variant of [7, Proposition 3.2(1.i)]. From now on, we denote by $\bar{f}$ the extension by zero outside $\Omega$ of a function $f \in L^{p}(\Omega)$.

Proposition 4.1. Let $1<q<+\infty$. Let $\rho: \mathbb{R}^{N} \rightarrow \mathbb{R}$ be a nonnegative continuous radial function with compact support, non-identically zero, and $\rho_{n}(x):=n^{N} \rho(n x)$. Let $S$ an open set, $S \subset \Omega$, and let $l \in L^{\infty}\left(\mathbb{R}^{N}\right)$ such that

$$
l(x)= \begin{cases}l(x)>0 & \text { a.e. in } S, \\ 0 & \text { a.e. in } \mathbb{R}^{N} \backslash S .\end{cases}
$$

Let us also assume that $l$ satisfies

$$
l^{\frac{1}{1-q}} \in L_{l o c}^{1}(S) .
$$

Let $\left\{f_{n}\right\}$ be a sequence of functions in $L^{q}(\Omega)$ such that

$$
\int_{\Omega} \int_{\Omega} \rho_{n}(y-x) l\left(\frac{x+y}{2}\right)\left|f_{n}(y)-f_{n}(x)\right|^{q} d x d y \leq M \frac{1}{n^{q}}
$$

and $\left\{f_{n}\right\}$ is weakly convergent in $L^{q}(S)$ to $f$.

Then, $l^{1 / q}|\nabla f| \in L^{q}(S),|\nabla f| \in L_{l o c}^{1}(S)$, and moreover

$$
\begin{gathered}
\lim _{n}\left[(\rho(z))^{1 / q}(l(w))^{1 / q} \chi_{\Omega}\left(w+\frac{1}{2 n}\right) \chi_{\Omega}\left(w-\frac{1}{2 n} z\right) \frac{\bar{f}_{n}\left(w+\frac{1}{2 n} z\right)-\bar{f}_{n}\left(w-\frac{1}{2 n} z\right)}{1 / n}\right]= \\
(\rho(z))^{1 / q} h(w, z)
\end{gathered}
$$

weakly in $L^{q}\left(\mathbb{R}^{N}\right) \times L^{q}\left(\mathbb{R}^{N}\right)$, with

$$
(\rho(z))^{1 / q} h(w, z)=(\rho(z))^{1 / q}(l(w))^{1 / q} z \cdot \nabla f(w) \quad \text { in } S \times \mathbb{R}^{N},
$$

and

$$
(\rho(z))^{1 / q} h(w, z)=0 \quad \text { in }\left(\mathbb{R}^{N} \backslash \bar{\Omega}\right) \times \mathbb{R}^{N} .
$$


Proof. Making the change of variables $y=x+\frac{1}{n} z, x=w-\frac{1}{2 n} z$, we rewrite (4.4) as

$$
\int_{\mathbb{R}^{N}} \int_{\mathbb{R}^{N}} \rho(z) l(w) \chi_{\Omega}^{\times}\left(w \pm \frac{1}{2 n} z\right)\left|\frac{\bar{f}_{n}\left(w+\frac{1}{2 n} z\right)-\bar{f}_{n}\left(w-\frac{1}{2 n} z\right)}{1 / n}\right|^{q} d w d z \leq M,
$$

where we use the notation $\chi_{\Omega}^{\times}\left(w \pm \frac{1}{2 n} z\right)=\chi_{\Omega}\left(w+\frac{1}{2 n} z\right) \chi_{\Omega}\left(w-\frac{1}{2 n} z\right)$. Therefore, up to a subsequence,

$$
(\rho(z))^{1 / q}(l(w))^{1 / q} \chi_{\Omega}^{\times}\left(w \pm \frac{1}{2 n} z\right) \frac{\bar{f}_{n}\left(w+\frac{1}{2 n} z\right)-\bar{f}_{n}\left(w-\frac{1}{2 n} z\right)}{1 / n} \rightarrow(\rho(z))^{1 / q} h(w, z)
$$

weakly in $L^{q}\left(\mathbb{R}^{N}\right) \times L^{q}\left(\mathbb{R}^{N}\right)$, and $(\rho(z))^{1 / q} h(w, z)=0$ in $\left(\mathbb{R}^{N} \backslash \bar{\Omega}\right) \times \mathbb{R}^{N}$.

If $\varphi \in C_{c}^{\infty}(\Omega), \operatorname{supp}(\varphi) \subset S$, taking

$$
\hat{\varphi}= \begin{cases}\frac{\varphi}{l^{1 / q}} & \text { in } S \\ 0 & \text { otherwise }\end{cases}
$$

which is an $L^{q^{\prime}}$-function since $l^{\frac{1}{1-q}} \in L_{l o c}^{1}(S)$, and $\psi \in C_{c}^{\infty}\left(\mathbb{R}^{N}\right)$, by (4.5), we obtain

$$
\begin{gathered}
\int_{\mathbb{R}^{N}} \int_{\Omega}(\rho(z))^{1 / q}(l(w))^{1 / q} \chi_{\Omega}^{\times}\left(w \pm \frac{1}{2 n} z\right) \frac{\bar{f}_{n}\left(w+\frac{1}{2 n} z\right)-\bar{f}_{n}\left(w-\frac{1}{2 n} z\right)}{1 / n} \hat{\varphi}(w) d w \psi(z) d z \\
\rightarrow \int_{\mathbb{R}^{N}} \int_{\Omega}(\rho(z))^{1 / q} h(w, z) \hat{\varphi}(w) d w \psi(z) d z
\end{gathered}
$$

That is,

$$
\begin{gathered}
\int_{\mathbb{R}^{N}} \int_{S}(\rho(z))^{1 / q} \chi_{\Omega}^{\times}\left(w \pm \frac{1}{2 n} z\right) \frac{\bar{f}_{n}\left(w+\frac{1}{2 n} z\right)-\bar{f}_{n}\left(w-\frac{1}{2 n} z\right)}{1 / n} \varphi(w) d w \psi(z) d z \\
\rightarrow \int_{\mathbb{R}^{N}} \int_{S}(\rho(z))^{1 / q}(l(w))^{-1 / q} h(w, z) \varphi(w) d w \psi(z) d z .
\end{gathered}
$$

Now, for $n$ large enough, $\rho(z)^{1 / q} \chi_{\Omega}^{\times}\left(w \pm \frac{1}{2 n} z\right)=\rho(z)^{1 / q}$ for all $z \in \mathbb{R}^{N}$ and all $w \in \operatorname{supp}(\varphi)$, therefore

$$
\begin{aligned}
\int_{\mathbb{R}^{N}} & \int_{S}(\rho(z))^{1 / q} \chi_{\Omega}^{\times}\left(w \pm \frac{1}{2 n} z\right) \frac{\bar{f}_{n}\left(w+\frac{1}{2 n} z\right)-\bar{f}_{n}\left(w-\frac{1}{2 n} z\right)}{1 / n} \varphi(w) d w \psi(z) d z \\
& =\int_{\mathbb{R}^{N}}(\rho(z))^{1 / q} \int_{S} \frac{\bar{f}_{n}\left(w+\frac{1}{2 n} z\right)-\bar{f}_{n}\left(w-\frac{1}{2 n} z\right)}{1 / n} \varphi(w) d w \psi(z) d z \\
& =-\int_{\mathbb{R}^{N}}(\rho(z))^{1 / q} \int_{S} f_{n}(w) \frac{\bar{\varphi}\left(w+\frac{1}{2 n} z\right)-\bar{\varphi}\left(w-\frac{1}{2 n} z\right)}{1 / n} d w \psi(z) d z
\end{aligned}
$$

Then, passing to the limit, on account of (4.6), we get

$$
\begin{aligned}
& \int_{\mathbb{R}^{N}}(\rho(z))^{1 / q} \int_{S}(l(w))^{-1 / q} h(w, z) \varphi(w) d w \psi(z) d z \\
& =-\int_{\mathbb{R}^{N}}(\rho(z))^{1 / q} \int_{S} f(w) z \cdot \nabla \varphi(w) d w \psi(z) d z .
\end{aligned}
$$


Consequently,

$$
\int_{S}(l(w))^{-1 / q} h(w, z) \varphi(w) d w=-\int_{S} f(w) z \cdot \nabla \varphi(w) d w \quad \forall z \in \operatorname{int}(\operatorname{supp}(J)) .
$$

From here, for $s$ small,

$$
\int_{S}(l(w))^{-1 / q} h\left(w, s e_{i}\right) \varphi(w) d w=-\int_{S} f(w) s \frac{\partial}{\partial w_{i}} \varphi(w) d w
$$

which implies, since $S$ is open, $|\nabla f| \in L_{l o c}^{1}(S)$ (using that $l^{\frac{1}{1-q}} \in L_{l o c}^{1}(S)$ together with Hölder's inequality), $l^{1 / q}|\nabla f| \in L^{q}(S)$ and $(\rho(z))^{1 / q} h(w, z)=(\rho(z))^{1 / q}(l(w))^{1 / q} z \cdot \nabla f(w)$ in $S \times \mathbb{R}^{N}$.

Proposition 4.2. Assume $p>1, J$ satisfies (HJ), and g satisfies (3.1) and (3.3). Then, for any $\phi \in L^{\infty}(\Omega)$, we have that

$$
\left(I+B_{p}^{J_{p, \varepsilon}, g}\right)^{-1} \phi \rightarrow\left(I+B_{p}^{g}\right)^{-1} \phi \quad \text { weakly in } L^{p}(\Omega) \text { as } \varepsilon \rightarrow 0 .
$$

Proof. For $\varepsilon>0$, let $u_{\varepsilon}=\left(I+B_{p}^{J_{p, \varepsilon}, g}\right)^{-1} \phi$. Then,

$$
\begin{aligned}
\int_{\Omega} u_{\varepsilon} v-\frac{C_{J, p}}{\varepsilon^{p+N}} \int_{\Omega} \int_{\Omega} J & \left(\frac{x-y}{\varepsilon}\right) g\left(\frac{x+y}{2}\right)\left|u_{\varepsilon}(y)-u_{\varepsilon}(x)\right|^{p-2} \times \\
& \times\left(u_{\varepsilon}(y)-u_{\varepsilon}(x)\right) d y v(x) d x=\int_{\Omega} \phi v
\end{aligned}
$$

for every $v \in L^{\infty}(\Omega)$. Moreover, $u_{\varepsilon} \ll \phi$. Changing variables, we get

$$
\begin{gathered}
-\frac{C_{J, p}}{\varepsilon^{p+N}} \int_{\Omega} \int_{\Omega} J\left(\frac{x-y}{\varepsilon}\right) g\left(\frac{x+y}{2}\right)\left|u_{\varepsilon}(y)-u_{\varepsilon}(x)\right|^{p-2}\left(u_{\varepsilon}(y)-u_{\varepsilon}(x)\right) d y v(x) d x \\
=\int_{\mathbb{R}^{N}} \int_{\mathbb{R}^{N}} \frac{C_{J, p}}{2} J(z) g(w) \chi_{\Omega}^{\times}\left(w \pm \frac{\varepsilon}{2} z\right)\left|\frac{\bar{u}_{\varepsilon}\left(w+\frac{\varepsilon}{2} z\right)-\bar{u}_{\varepsilon}\left(w-\frac{\varepsilon}{2} z\right)}{\varepsilon}\right|^{p-2} \times \\
\times \frac{\bar{u}_{\varepsilon}\left(w+\frac{\varepsilon}{2} z\right)-\bar{u}_{\varepsilon}\left(w-\frac{\varepsilon}{2} z\right)}{\varepsilon} \frac{\bar{v}\left(w+\frac{\varepsilon}{2} z\right)-\bar{v}\left(w-\frac{\varepsilon}{2} z\right)}{\varepsilon} d w d z
\end{gathered}
$$

where $\chi_{\Omega}^{\times}\left(w \pm \frac{\varepsilon}{2} z\right)=\chi_{\Omega}\left(w+\frac{\varepsilon}{2} z\right) \chi_{\Omega}\left(w-\frac{\varepsilon}{2} z\right)$. So we can rewrite (4.8) as

$$
\begin{aligned}
& \int_{\Omega} \phi(x) v(x) d x-\int_{\Omega} u_{\varepsilon}(x) v(x) d x \\
&=\int_{\mathbb{R}^{N}} \int_{\mathbb{R}^{N}} \frac{C_{J, p}}{2} J(z) g(w) \chi_{\Omega}^{\times}\left(w \pm \frac{\varepsilon}{2} z\right)\left|\frac{\bar{u}_{\varepsilon}\left(w+\frac{\varepsilon}{2} z\right)-\bar{u}_{\varepsilon}\left(w-\frac{\varepsilon}{2} z\right)}{\varepsilon}\right|^{p-2} \times \\
& \times \frac{\bar{u}_{\varepsilon}\left(w+\frac{\varepsilon}{2} z\right)-\bar{u}_{\varepsilon}\left(w-\frac{\varepsilon}{2} z\right)}{\varepsilon} \frac{\bar{v}\left(w+\frac{\varepsilon}{2} z\right)-\bar{v}\left(w-\frac{\varepsilon}{2} z\right)}{\varepsilon} d w d z .
\end{aligned}
$$

We will see there exists a sequence $\varepsilon_{n} \rightarrow 0$ such that $u_{\varepsilon_{n}} \rightarrow u$ weakly in $L^{p}(\Omega), u \in W_{g}^{1, p}(\Omega) \cap$ $L^{\infty}(\Omega)$, a solution of

$$
\int_{\Omega} u v+\int_{S} g|\nabla u|^{p-2} \nabla u \cdot \nabla v=\int_{\Omega} \phi v \quad \text { for every } v \in W^{1, p}(\Omega) \cap L^{\infty}(\Omega),
$$

that is, $u=\left(I+B_{p}^{g}\right)^{-1} \phi$. 
Since $u_{\varepsilon} \ll \phi$, there exists a sequence $\varepsilon_{n} \rightarrow 0$ such that

$$
u_{\varepsilon_{n}} \rightarrow u, \quad \text { weakly in } L^{p}(\Omega), \quad u \ll \phi .
$$

Observe that also $\left\|u_{\varepsilon_{n}}\right\|_{L^{\infty}(\Omega)},\|u\|_{L^{\infty}(\Omega)} \leq\|\phi\|_{L^{\infty}(\Omega)}$. Taking $\varepsilon=\varepsilon_{n}$ and $v=u_{\varepsilon_{n}}$ in (4.10), we get

$$
\begin{aligned}
& \int_{\Omega} \int_{\Omega} \frac{1}{2} \frac{C_{J, p}}{\varepsilon_{n}} J\left(\frac{x-y}{\varepsilon_{n}}\right) g\left(\frac{x+y}{2}\right)\left|\frac{u_{\varepsilon_{n}}(y)-u_{\varepsilon_{n}}(x)}{\varepsilon_{n}}\right|^{p} d x d y \\
& =\int_{\mathbb{R}^{N}} \int_{\mathbb{R}^{N}} \frac{C_{J, p}}{2} J(z) g(w) \chi_{\Omega}^{\times}\left(w \pm \frac{\varepsilon_{n}}{2} z\right)\left|\frac{\bar{u}_{\varepsilon_{n}}\left(w+\frac{\varepsilon_{n}}{2} z\right)-\bar{u}_{\varepsilon}\left(w-\frac{\varepsilon_{n}}{2} z\right)}{\varepsilon_{n}}\right|^{p} d w d z \leq M .
\end{aligned}
$$

Therefore, by Proposition 4.1, $u \in W_{g}^{1, p}(\Omega)$ and

$$
\left(\frac{C_{J, p}}{2} J(z)\right)^{1 / p}(g(w))^{1 / p} \chi_{\Omega}^{\times}\left(w \pm \frac{\varepsilon}{2} z\right) \frac{\bar{u}_{\varepsilon_{n}}\left(w+\frac{\varepsilon_{n}}{2} z\right)-\bar{u}_{\varepsilon_{n}}\left(w-\frac{\varepsilon_{n}}{2} z\right)}{\varepsilon_{n}} \rightarrow\left(\frac{C_{J, p}}{2} J(z)\right)^{1 / p} h(w, z)
$$

weakly in $L^{p}\left(\mathbb{R}^{N}\right) \times L^{p}\left(\mathbb{R}^{N}\right)$ with $(J(z))^{1 / p} h(w, z)=(J(z))^{1 / p}(g(w))^{1 / p} z \cdot \nabla u(w)$ in $S \times \mathbb{R}^{N}$ and $(J(z))^{1 / p} h(w, z)=0$ in $\left(\mathbb{R}^{N} \backslash \bar{\Omega}\right) \times \mathbb{R}^{N}$. Moreover, we can also assume that

$$
\begin{aligned}
J(z)^{1 / p^{\prime}} g(w)^{1 / p^{\prime}} & \chi_{\Omega}^{\times}\left(w \pm \frac{\varepsilon_{n}}{2} z\right)\left|\frac{\bar{u}_{\varepsilon_{n}}\left(w+\frac{\varepsilon_{n}}{2} z\right)-\bar{u}_{\varepsilon_{n}}\left(w-\frac{\varepsilon_{n}}{2} z\right)}{\varepsilon_{n}}\right|^{p-2} \\
& \times \frac{\bar{u}_{\varepsilon_{n}}\left(w+\frac{\varepsilon_{n}}{2} z\right)-\bar{u}_{\varepsilon_{n}}\left(w-\frac{\varepsilon_{n}}{2} z\right)}{\varepsilon_{n}} \rightarrow J(z)^{1 / p^{\prime}} \chi(w, z)
\end{aligned}
$$

weakly in $L^{p^{\prime}}\left(\mathbb{R}^{N}\right) \times L^{p^{\prime}}\left(\mathbb{R}^{N}\right)$, with $J(z)^{1 / p^{\prime}} \chi(w, z)=0$ in $\left(\mathbb{R}^{N} \backslash \bar{\Omega}\right) \times \mathbb{R}^{N}$. Therefore, passing to the limit in (4.10) for $\varepsilon=\varepsilon_{n}$, we get

$$
\int_{\Omega} u v+\int_{\mathbb{R}^{N}} \int_{S} \frac{C_{J, p}}{2} J(z) g(w)^{1 / p} \chi(w, z) z \cdot \nabla v(w) d w d z=\int_{\Omega} \phi v
$$

for every $v$ smooth and, by approximation, for every $v \in W_{g, S}^{1, p}(\Omega)$. Then, also

$$
\int_{\Omega} u^{2}+\int_{\mathbb{R}^{N}} \int_{S} \frac{C_{J, p}}{2} J(z) g(w)^{1 / p} \chi(w, z) z \cdot \nabla u(w) d w d z=\int_{\Omega} \phi u .
$$

Let us see now that, for every $v \in W_{g, S}^{1, p}(\Omega)$,

$$
\int_{\mathbb{R}^{N}} \int_{S} \frac{C_{J, p}}{2} J(z) g(w)^{1 / p} \chi(w, z) z \cdot \nabla v(x) d w d z=\int_{S} g|\nabla u|^{p-2} \nabla u \cdot \nabla v .
$$

In fact, taking $v=u_{\varepsilon_{n}}$ in (4.8), taking limits and considering (4.15), we get

$$
\begin{aligned}
\limsup _{n} & \int_{\mathbb{R}^{N}} \int_{\mathbb{R}^{N}} \frac{C_{J, p}}{2} J(z) g(w) \chi_{\Omega}^{\times}\left(w \pm \frac{\varepsilon_{n}}{2} z\right)\left|\frac{\bar{u}_{\varepsilon_{n}}\left(w+\frac{\varepsilon_{n}}{2} z\right)-\bar{u}_{\varepsilon_{n}}\left(w-\frac{\varepsilon_{n}}{2} z\right)}{\varepsilon_{n}}\right|^{p} d x d z \\
& \leq \int_{\Omega} \phi u-\int_{\Omega} u u=\int_{\mathbb{R}^{N}} \int_{S} \frac{C_{J, p}}{2} J(z) g(w)^{1 / p} \chi(x, z) z \cdot \nabla u(x) d x d z .
\end{aligned}
$$


Now, by the monotonicity Lemma 2.5 , for every $\rho$ smooth,

$$
\begin{aligned}
& -\int_{\Omega} \int_{\Omega} J\left(\frac{x-y}{\varepsilon_{n}}\right) g\left(\frac{x+y}{2}\right)|\rho(y)-\rho(x)|^{p-2}(\rho(y)-\rho(x)) d y\left(u_{\varepsilon_{n}}(x)-\rho(x)\right) d x \\
& \leq-\int_{\Omega} \int_{\Omega} J\left(\frac{x-y}{\varepsilon_{n}}\right) g\left(\frac{x+y}{2}\right)\left|u_{\varepsilon_{n}}(y)-u_{\varepsilon_{n}}(x)\right|^{p-2}\left(u_{\varepsilon_{n}}(y)-u_{\varepsilon_{n}}(x)\right) d y\left(u_{\varepsilon_{n}}(x)-\rho(x)\right) d x .
\end{aligned}
$$

Using the change of variable (4.9) and taking limits, on account of (4.13) and (4.17), we obtain for every $\rho$ smooth,

$$
\begin{aligned}
& \int_{\mathbb{R}^{N}} \int_{S} \frac{C_{J, p}}{2} J(z) g(w)|z \cdot \nabla \rho(w)|^{p-2} z \cdot \nabla \rho(w) z \cdot(\nabla u(w)-\nabla \rho(w)) d w d z, \\
& \leq \int_{\mathbb{R}^{N}} \int_{S} \frac{C_{J, p}}{2} J(z) g(w)^{1 / p} \chi(w, z) z \cdot(\nabla u(w)-\nabla \rho(w)) d w d z .
\end{aligned}
$$

Now, by approximation, we can take in (4.18), $\rho=u \pm \lambda v, \lambda>0, v \in W_{g, S}^{1, p}(\Omega)$. So, letting $\lambda \rightarrow 0$, we get

$$
\begin{aligned}
& \int_{\mathbb{R}^{N}} \int_{S} \frac{C_{J, p}}{2} J(z) g(w)^{1 / p^{\prime}} \chi(w, z) z \cdot \nabla v(w) d w d z \\
& =\int_{\mathbb{R}^{N}} \int_{S} \frac{C_{J, p}}{2} J(z) g(w)|z \cdot \nabla u(w)|^{p-2}(z \cdot \nabla u(w))(z \cdot \nabla v(w)) d w d z .
\end{aligned}
$$

Consequently,

$$
\begin{aligned}
& \int_{\mathbb{R}^{N}} \int_{S} \frac{C_{J, p}}{2} J(z) g(w)^{1 / p} \chi(w, z) z \cdot \nabla v(w) d w d z \\
&=C_{J, p} \int_{S} g(w) \mathbf{a}(\nabla u(w)) \cdot \nabla v(w) d w, \quad \text { for every } v \in W_{g, S}^{1, p}(\Omega),
\end{aligned}
$$

where

$$
\mathbf{a}_{j}(\xi)=C_{J, p} \int_{\mathbb{R}^{N}} \frac{1}{2} J(z)|z \cdot \xi|^{p-2} z \cdot \xi z_{j} d z .
$$

Then, since $\mathbf{a}(\xi)=|\xi|^{p-2} \xi$ (see [7] for the details), (4.16) is true.

Theorem 4.3. Assume $p>1, J$ satisfies (HJ) and $J(x) \geq J(y)$ if $|x| \leq|y|$, and $g$ satisfies (3.1) and (3.3). Assume also $g$ is lower semicontinuous. Then, for any $\phi \in L^{\infty}(\Omega)$, we have that

$$
\left(I+B_{p}^{J_{p, \varepsilon}, g}\right)^{-1} \phi \rightarrow\left(I+B_{p}^{g}\right)^{-1} \phi \quad \text { in } L^{p}(\Omega) \text { as } \varepsilon \rightarrow 0 .
$$

Proof. For each $m \in \mathbb{N}$, let the open sets $S_{m}=\{x \in \Omega: \operatorname{dist}(x, \partial S)>1 / m\}$. We have that $S=\cup_{m} S_{m}$, there exists $\alpha_{m}>0$ such that $g(x) \geq \alpha_{m}>0$ for every $x \in S_{m}$, and there exists a finite number of balls $B_{i}$ covering $S_{m}$, with $B_{i} \subset S_{m+1}$.

Let $\varepsilon_{n}$ a subsequence converging to 0 . We can suppose that such sequence, or a subsequence if necessary, satisfies (4.12), then, in each ball $B_{i}$,

$$
\int_{B_{i}} \int_{B_{i}} \frac{1}{2} \frac{C_{J, p}}{\varepsilon_{n}^{N}} J\left(\frac{x-y}{\varepsilon_{n}}\right) g\left(\frac{x+y}{2}\right)\left|\frac{u_{\varepsilon_{n}}(y)-u_{\varepsilon_{n}}(x)}{\varepsilon_{n}}\right|^{p} d x d y \leq M,
$$

and also

$$
\int_{B_{i}} \int_{B_{i}} \frac{1}{2} \frac{C_{J, p}}{\varepsilon_{n} N} J\left(\frac{x-y}{\varepsilon_{n}}\right)\left|\frac{u_{\varepsilon_{n}}(y)-u_{\varepsilon_{n}}(x)}{\varepsilon_{n}}\right|^{p} d x d y \leq M / \alpha_{m+1} .
$$


Therefore, by [7, Proposition 3.2(2.i)] (see also [17, Theorem 4]), taking into account (4.7),

$$
\left(I+B_{p}^{J_{p, \varepsilon_{n}}, g}\right)^{-1} \phi \rightarrow\left(I+B_{p}^{g}\right)^{-1} \phi \quad \text { a.e. in } \Omega .
$$

Now, since in fact $\left\{\left(I+B_{p}^{J_{p, \varepsilon_{n}}, g}\right)^{-1} \phi\right\}$ is bounded in $L^{\infty}(\Omega)$ the result follows.

From the above theorem, by standard results of the Nonlinear Semigroup Theory (see [20] and $[16]$ ), we obtain the following result.

Theorem 4.4. Let $p>1$. Assume $J$ satisfies (HJ) and $J(x) \geq J(y)$ if $|x| \leq|y|$, and $g$ satisfies (3.1) and (3.3). Let $T>0$ and $u_{0} \in L^{p}(\Omega)$. Let $u_{\varepsilon}$ the unique solution of $P_{p}^{J_{p, \varepsilon}, g}\left(u_{0}\right)$ and $u$ the unique entropy solution of $N_{p}^{g}\left(u_{0}\right)$. Then

$$
\lim _{\varepsilon \rightarrow 0} \sup _{t \in[0, T]}\left\|u_{\varepsilon}(t, \cdot)-u(t, \cdot)\right\|_{L^{p}(\Omega)}=0 .
$$

Proof. Since $B_{p}^{J, g}$ and $\mathcal{B}_{p}^{g} \cap\left(L^{p}(\Omega) \times L^{p}(\Omega)\right)$ are $m$-completely accretive in $L^{p}(\Omega)$, to get the result it is enough to see that $\left(I+B_{p}^{J_{p, \varepsilon}, g}\right)^{-1} \phi \rightarrow\left(I+B_{p}^{g}\right)^{-1} \phi$ in $L^{p}(\Omega)$ as $\varepsilon \rightarrow 0$ for any $\phi \in L^{\infty}(\Omega)$, which follows by Theorem 4.3.

5. EXISTENCE AND UNIQUENESS OF SOLUTIONS FOR THE NONLOCAL PROBLEM.

THE CASE $p=1$

This section deals with the existence and uniqueness of solutions for the nonlocal problem

$$
P_{1}^{J, g}\left(u_{0}\right) \quad\left\{\begin{array}{l}
u_{t}(t, x)=\int_{\Omega} J(x-y) g\left(\frac{x+y}{2}\right) \frac{u(t, y)-u(t, x)}{|u(t, y)-u(t, x)|} d y . \\
u(x, 0)=u_{0}(x) .
\end{array}\right.
$$

First, let us introduce what we will understand by a solution.

Definition 5.1. A solution of $P_{1}^{J, g}\left(u_{0}\right)$ in $[0, T]$ is a function $u \in W^{1,1}\left(0, T ; L^{1}(\Omega)\right)$ which satisfies $u(0, x)=u_{0}(x)$ a.e. $x \in \Omega$ and

$$
\left.u_{t}(t, x)=\int_{\Omega} J(x-y) g\left(\frac{x+y}{2}\right) h(t, x, y) d y \quad \text { a.e. in } \quad\right] 0, T[\times \Omega,
$$

for some $h \in L^{\infty}\left(0, T ; L^{\infty}(\Omega \times \Omega)\right)$ with $\|h\|_{\infty} \leq 1$ such that $h(t, x, y)=-h(t, y, x)$ and

$$
J(x-y) g\left(\frac{x+y}{2}\right) h(t, x, y) \in J(x-y) g\left(\frac{x+y}{2}\right) \operatorname{sign}(u(t, y)-u(t, x)) .
$$

Here $\operatorname{sign}(\cdot)$ is the multivalued function given by

$$
\operatorname{sign}(r)= \begin{cases}-1 & \text { if } r<0 \\ {[-1,1]} & \text { if } r=0 \\ 1 & \text { if } r>0\end{cases}
$$

As in the case $p>1$, to prove the existence and uniqueness of solutions of $P_{1}^{J}\left(u_{0}\right)$ we use the Nonlinear Semigroup Theory, so we start introducing the following operator in $L^{1}(\Omega)$. 
Definition 5.2. Let $J$ satisfies (HJ), $g \in L^{\infty}\left(\mathbb{R}^{N}\right), g \geq 0$ a.e. We define the operator $B_{1}^{J, g}$ in $L^{1}(\Omega) \times L^{1}(\Omega)$ by $\hat{u} \in B_{1}^{J, g} u$ if and only if $u, \hat{u} \in L^{1}(\Omega)$, there exists $h \in L^{\infty}(\Omega \times \Omega)$, $h(x, y)=-h(y, x)$ for almost all $(x, y) \in \Omega \times \Omega,\|h\|_{\infty} \leq 1$,

$$
\hat{u}(x)=-\int_{\Omega} J(x-y) g\left(\frac{x+y}{2}\right) h(x, y) d y, \quad \text { a.e. } x \in \Omega
$$

and

$$
J(x-y) g\left(\frac{x+y}{2}\right) h(x, y) \in J(x-y) g\left(\frac{x+y}{2}\right) \operatorname{sign}(u(y)-u(x)),
$$

a.e. $(x, y) \in \Omega \times \Omega$.

\section{Remark 5.3.}

1. It is not difficult to see that (5.1) is equivalent to

$-\int_{\Omega} \int_{\Omega} J(x-y) g\left(\frac{x+y}{2}\right) h(x, y) d y u(x) d x=\frac{1}{2} \int_{\Omega} \int_{\Omega} J(x-y) g\left(\frac{x+y}{2}\right)|u(y)-u(x)| d y d x$,

2. $L^{1}(\Omega)=\operatorname{Dom}\left(B_{1}^{J, g}\right)$ and $B_{1}^{J, g}$ is closed in $L^{1}(\Omega) \times L^{1}(\Omega)$.

3. $B_{1}^{J, g}$ is positively homogeneous of degree zero, that is, if $\hat{u} \in B_{1}^{J, g} u$ and $\lambda>0$ then $\lambda \hat{u} \in$ $B_{1}^{J, g}(\lambda u)$.

Theorem 5.4. The operator $B_{1}^{J, g}$ is completely accretive and satisfies $L^{\infty}(\Omega) \subset \operatorname{Ran}\left(I+B_{1}^{J, g}\right)$.

Proof. Let $\hat{u}_{i} \in B_{1}^{J, g} u_{i}, i=1,2$. Then there exists $h_{i} \in L^{\infty}(\Omega \times \Omega),\left\|h_{i}\right\|_{\infty} \leq 1, h_{i}(x, y)=$ $-h_{i}(y, x), J(x-y) g\left(\frac{x+y}{2}\right) h_{i}(x, y) \in J(x-y) g\left(\frac{x+y}{2}\right) \operatorname{sign}\left(u_{i}(y)-u_{i}(x)\right)$ for almost all $(x, y) \in$ $\Omega \times \Omega$, such that

$$
\hat{u}_{i}(x)=-\int_{\Omega} J(x-y) g\left(\frac{x+y}{2}\right) h_{i}(x, y) d y, \quad \text { a.e. } x \in \Omega,
$$

for $i=1,2$. Given $q \in P_{0}$, we have

$$
\begin{aligned}
& \int_{\Omega}\left(\hat{u}_{1}(x)-\hat{u}_{2}(x)\right) q\left(u_{1}(x)-u_{2}(x)\right) d x \\
& =\frac{1}{2} \int_{\Omega} \int_{\Omega} J(x-y) g\left(\frac{x+y}{2}\right)\left(g_{1}(x, y)-g_{2}(x, y)\right)\left(q\left(u_{1}(y)-u_{2}(y)\right)-q\left(u_{1}(x)-u_{2}(x)\right)\right) d x d y .
\end{aligned}
$$

Now, by the mean value Theorem

$$
\begin{aligned}
& J(x-y) g\left(\frac{x+y}{2}\right)\left(h_{1}(x, y)-h_{2}(x, y)\right)\left[q\left(u_{1}(y)-u_{2}(y)\right)-q\left(u_{1}(x)-u_{2}(x)\right)\right] \\
& =J(x-y) g\left(\frac{x+y}{2}\right)\left(h_{1}(x, y)-h_{2}(x, y)\right) q^{\prime}(\xi)\left[\left(u_{1}(y)-u_{2}(y)\right)-\left(u_{1}(x)-u_{2}(x)\right)\right] \\
& =J(x-y) g\left(\frac{x+y}{2}\right) q^{\prime}(\xi)\left[h_{1}(x, y)\left(u_{1}(y)-u_{1}(x)\right)-h_{1}(x, y)\left(u_{2}(y)-u_{2}(x)\right)\right] \\
& \quad-J(x-y) g\left(\frac{x+y}{2}\right) q^{\prime}(\xi)\left[h_{2}(x, y)\left(u_{1}(y)-u_{1}(x)\right)-h_{2}(x, y)\left(u_{2}(y)-u_{2}(x)\right)\right] \geq 0,
\end{aligned}
$$

since

$$
J(x-y) g\left(\frac{x+y}{2}\right) h_{i}(x, y)\left(u_{i}(y)-u_{i}(x)\right)=J(x-y) g\left(\frac{x+y}{2}\right)\left|u_{i}(y)-u_{i}(x)\right|, \quad i=1,2,
$$


and

$$
-J(x-y) g\left(\frac{x+y}{2}\right) h_{i}(x, y)\left(u_{j}(y)-u_{j}(x)\right) \geq-J(x-y) g\left(\frac{x+y}{2}\right)\left|u_{j}(y)-u_{j}(x)\right|, \quad i \neq j,
$$

we get

$$
\int_{\Omega}\left(\hat{u}_{1}(x)-\hat{u}_{2}(x)\right) q\left(u_{1}(x)-u_{2}(x)\right) d x \geq 0,
$$

from where it follows that $B_{1}^{J, g}$ is a completely accretive operator.

To show that $B_{1}^{J, g}$ satisfies the range condition, let us see that for any $\phi \in L^{\infty}(\Omega)$,

$$
\lim _{p \rightarrow 1+}\left(I+B_{p}^{J, g}\right)^{-1} \phi=\left(I+B_{1}^{J, g}\right)^{-1} \phi \quad \text { weakly in } L^{1}(\Omega) .
$$

Let $\phi \in L^{\infty}(\Omega)$. For $1<p<+\infty$, by Theorem 2.6, there is $u_{p}$ such that $u_{p}=\left(I+B_{p}^{J, g}\right)^{-1} \phi$, that is,

$$
u_{p}(x)-\int_{\Omega} J(x-y) g\left(\frac{x+y}{2}\right)\left|u_{p}(y)-u_{p}(x)\right|^{p-2}\left(u_{p}(y)-u_{p}(x)\right) d y=\phi(x) \text { a.e. } x \in \Omega .
$$

Thus, for every $v \in L^{\infty}(\Omega)$, we can write

$$
\int_{\Omega} u_{p} v-\int_{\Omega} \int_{\Omega} J(x-y) g\left(\frac{x+y}{2}\right)\left|u_{p}(y)-u_{p}(x)\right|^{p-2}\left(u_{p}(y)-u_{p}(x)\right) d y v(x) d x=\int_{\Omega} \phi v .
$$

Since $u_{p} \ll \phi$, by Proposition 2.1, we have that there exists a sequence $p_{n} \rightarrow 1$ such that

$$
u_{p_{n}} \rightarrow u \quad \text { weakly in } L^{2}(\Omega), \quad u \ll \phi .
$$

Observe that $\left\|u_{p_{n}}\right\|_{L^{\infty}(\Omega)},\|u\|_{L^{\infty}(\Omega)} \leq\|\phi\|_{L^{\infty}(\Omega)}$. Now, since

$$
|| u_{p_{n}}(y)-\left.u_{p_{n}}(x)\right|^{p_{n}-2}\left(u_{p_{n}}(y)-u_{p_{n}}(x)\right) \mid \leq\left(2\|\phi\|_{\infty}\right)^{p_{n}-1},
$$

there exists $h(x, y)$ such that

$$
\left|u_{p_{n}}(y)-u_{p_{n}}(x)\right|^{p_{n}-2}\left(u_{p_{n}}(y)-u_{p_{n}}(x)\right) \rightarrow h(x, y),
$$

weakly in $L^{1}(\Omega \times \Omega), g(x, y)=-g(y, x)$ for almost all $(x, y) \in \Omega \times \Omega$, and $\|h\|_{\infty} \leq 1$.

Therefore, passing to the limit in (5.2) for $p=p_{n}$, we get

$$
\int_{\Omega} u v-\int_{\Omega} \int_{\Omega} J(x-y) g\left(\frac{x+y}{2}\right) h(x, y) d y v(x) d x=\int_{\Omega} \phi v
$$

for every $v \in L^{\infty}(\Omega)$, and consequently we get

$$
u(x)-\int_{\Omega} J(x-y) g\left(\frac{x+y}{2}\right) h(x, y) d y=\phi(x) \quad \text { a.e. } x \in \Omega .
$$

Then, to finish the proof we have to show that

$$
-\int_{\Omega} \int_{\Omega} J(x-y) g\left(\frac{x+y}{2}\right) h(x, y) d y u(x) d x=\frac{1}{2} \int_{\Omega} \int_{\Omega} J(x-y) g\left(\frac{x+y}{2}\right)|u(y)-u(x)| d y d x .
$$

In fact, by (5.2),

$$
\frac{1}{2} \int_{\Omega} \int_{\Omega} J(x-y)\left|u_{p_{n}}(y)-u_{p_{n}}(x)\right|^{p_{n}} d y d x=\int_{\Omega} \phi u_{p_{n}}-\int_{\Omega} u_{p_{n}} u_{p_{n}}
$$


so, using (5.3) and (5.4) with $v=u$, we get

$$
\begin{aligned}
& \limsup _{n \rightarrow+\infty} \frac{1}{2} \int_{\Omega} \int_{\Omega} J(x-y) g\left(\frac{x+y}{2}\right)\left|u_{p_{n}}(y)-u_{p_{n}}(x)\right|^{p_{n}} d y d x \\
& \leq \int_{\Omega} \phi u-\int_{\Omega} u u=-\int_{\Omega} \int_{\Omega} J(x-y) g\left(\frac{x+y}{2}\right) h(x, y) d y u(x) d x .
\end{aligned}
$$

Now, by the monotonicity Lemma 2.5 , for all $\rho \in L^{\infty}(\Omega)$,

$$
\begin{aligned}
& -\int_{\Omega} \int_{\Omega} J(x-y) g\left(\frac{x+y}{2}\right)|\rho(y)-\rho(x)|^{p_{n}-2}(\rho(y)-\rho(x)) d y\left(u_{p_{n}}(x)-\rho(x)\right) d x \\
& \leq-\int_{\Omega} \int_{\Omega} J(x-y) g\left(\frac{x+y}{2}\right)\left|u_{p_{n}}(y)-u_{p_{n}}(x)\right|^{p_{n}-2}\left(u_{p_{n}}(y)-u_{p_{n}}(x)\right) d y\left(u_{p_{n}}(x)-\rho(x)\right) d x .
\end{aligned}
$$

Therefore, taking limits, we obtain

$$
\begin{gathered}
-\int_{\Omega} \int_{\Omega} J(x-y) g\left(\frac{x+y}{2}\right) \operatorname{sign}_{0}(\rho(y)-\rho(x)) d y(u(x)-\rho(x)) d x \\
\leq-\int_{\Omega} \int_{\Omega} J(x-y) g\left(\frac{x+y}{2}\right) h(x, y) d y(u(x)-\rho(x)) d x .
\end{gathered}
$$

Taking now, $\rho=u \pm \lambda u, \lambda>0$, and letting $\lambda \rightarrow 0$, we get (5.5), and the proof is finished.

Theorem 5.5. For every initial datum $u_{0} \in L^{1}(\Omega)$ and ant $T>0$ the problem $P_{1}^{J, g}\left(u_{0}\right)$ has a unique solution in $(0, T)$ and, moreover, an $L^{1}$-contraction principle holds for such solutions.

Proof. As a consequence of the above results, we have that the abstract Cauchy problem

$$
\left\{\begin{array}{l}
\left.u^{\prime}(t)+B_{1}^{J, g} u(t) \ni 0, \quad t \in\right] 0, T[, \\
u(0)=u_{0}
\end{array}\right.
$$

has a unique mild solution $u$ for every initial datum $u_{0} \in L^{1}(\Omega)$ and $T>0$ (see [16]). Moreover, due to the complete accretivity and the homogeneity of the operator $B_{1}^{J, g}$, the mild solution of (5.6) is a strong solution ([15]) and, so, a solution of $P_{1}^{J, g}\left(u_{0}\right)$.

\section{The Local Problem for $p=1$}

Let $\Omega \subset \mathbb{R}^{N}$ a bounded domain and $0 \leq g \in L^{\infty}(\Omega)$. In this section we are interested in the following local diffusion equation with homogeneous Neumann boundary condition,

$$
N_{1}^{g}\left(u_{0}\right) \begin{cases}u_{t}=\operatorname{div}\left(g \frac{D u}{|D u|}\right) & \text { in }] 0, T[\times \Omega, \\ g \frac{D u}{|D u|} \cdot \eta=0 & \text { on }] 0, T[\times \partial \Omega, \\ u(x, 0)=u_{0}(x) & \text { in } \Omega,\end{cases}
$$

where $\eta$ is the unit outward normal on $\partial \Omega$.

Due to the linear growth condition on the Lagrangian, the natural energy space to study problem $N_{1}^{g}\left(u_{0}\right)$ is the space of functions of bounded variation. Let us recall several facts concerning functions of bounded variation (for further information concerning functions of bounded variation we refer to [38], [51] or [2]). 
A function $u \in L^{1}(\Omega)$ whose partial derivatives in the sense of distributions are measures with finite total variation in $\Omega$ is called a function of bounded variation. The class of such functions will be denoted by $B V(\Omega)$. Thus $u \in B V(\Omega)$ if and only if there are Radon measures $\mu_{1}, \ldots, \mu_{N}$ defined in $\Omega$ with finite total mass in $\Omega$ and

$$
\int_{\Omega} u D_{i} \varphi d x=-\int_{\Omega} \varphi d \mu_{i}
$$

for all $\varphi \in C_{0}^{\infty}(\Omega), i=1, \ldots, N$. Thus the gradient of $u$ is a vector valued measure with finite total variation

$$
|D u|=\sup \left\{\int_{\Omega} u \operatorname{div} \varphi d x: \varphi \in C_{0}^{\infty}\left(\Omega, \mathbb{R}^{N}\right),|\varphi(x)| \leq 1 \text { for } x \in \Omega\right\} .
$$

The space $B V(\Omega)$ is endowed with the norm $\|u\|_{B V}=\|u\|_{L^{1}(\Omega)}+|D u|$. For $u \in B V(\Omega)$, the gradient $D u$ is a Radon measure that decomposes into its absolutely continuous and singular parts $D u=D^{a} u+D^{s} u$. Then $D^{a} u=\nabla u \mathcal{L}^{N}$ where $\nabla u$ is the Radon-Nikodym derivative of the measure $D u$ with respect to the Lebesgue measure $\mathcal{L}^{N}$.

We shall need several results from [10] (see also [4]). Following [10], let

$$
X_{p}(\Omega)=\left\{\mathbf{z} \in L^{\infty}\left(\Omega, \mathbb{R}^{N}\right): \operatorname{div}(\mathbf{z}) \in L^{p}(\Omega)\right\}, \quad 1 \leq p \leq N .
$$

If $\mathbf{z} \in X_{p}(\Omega)$ and $w \in B V(\Omega) \cap L^{p^{\prime}}(\Omega)$ we define the functional $(\mathbf{z}, D w): C_{0}^{\infty}(\Omega) \rightarrow \mathbb{R}$ by the formula

$$
\langle(\mathbf{z}, D w), \varphi\rangle=-\int_{\Omega} w \varphi \operatorname{div}(\mathbf{z}) d x-\int_{\Omega} w \mathbf{z} \cdot \nabla \varphi d x
$$

Then $(\mathbf{z}, D w)$ is a Radon measure in $\Omega$,

$$
\int_{\Omega}(\mathbf{z}, D w)=\int_{\Omega} \mathbf{z} \cdot \nabla w d x \quad \forall w \in W^{1,1}(\Omega) \cap L^{\infty}(\Omega)
$$

and

for any Borel set $B \subseteq \Omega$.

$$
\left|\int_{B}(\mathbf{z}, D w)\right| \leq \int_{B}|(\mathbf{z}, D w)| \leq\|\mathbf{z}\|_{\infty} \int_{B}|D w|
$$

In [10], a weak trace on $\partial \Omega$ of the normal component of $\mathbf{z} \in X_{p}(\Omega)$ is defined. Concretely, it is proved that there exists a linear operator $\gamma: X_{p}(\Omega) \rightarrow L^{\infty}(\partial \Omega)$ such that $\|\gamma(\mathbf{z})\|_{\infty} \leq\|\mathbf{z}\|_{\infty}$ and $\gamma(\mathbf{z})(x)=\mathbf{z}(x) \cdot \nu(x)$ for all $x \in \partial \Omega$ if $\mathbf{z} \in C^{1}\left(\bar{\Omega}, \mathbb{R}^{N}\right)$. We shall denote $\gamma(\mathbf{z})(x)$ by $[\mathbf{z}, \nu](x)$. Moreover, the following Green's formula, relating the function $[\mathbf{z}, \nu]$ and the measure $(\mathbf{z}, D w)$, for $\mathbf{z} \in X_{p}(\Omega)$ and $w \in B V(\Omega) \cap L^{p^{\prime}}(\Omega)$, is established:

$$
\int_{\Omega} w \operatorname{div}(\mathbf{z}) d x+\int_{\Omega}(\mathbf{z}, D w)=\int_{\partial \Omega}[\mathbf{z}, \nu] w d \mathcal{H}^{N-1} .
$$

To define the differential operator $\operatorname{div}\left(g \frac{D u}{|D u|}\right)$ we need to recall the concept of total variation with respect to an anisotropy (see [1], [14] and [24]). We say that a function $\phi: \Omega \times \mathbb{R}^{N} \rightarrow[0, \infty$ ) is a metric integrand if $\phi$ is a Borel function satisfying the conditions

$$
\begin{aligned}
& \text { for a.e. } x \in \Omega \text {, the map } \xi \in \mathbb{R}^{N} \rightarrow \phi(x, \xi) \text { is convex, } \\
& \phi(x, t \xi)=|t| \phi(x, \xi) \quad \forall x \in \Omega, \quad \forall \xi \in \mathbb{R}^{N}, \quad \forall t \in \mathbb{R},
\end{aligned}
$$


and there exists a constant $\Gamma>0$ such that

$$
0 \leq \phi(x, \xi) \leq \Gamma\|\xi\| \quad \forall x \in \Omega, \quad \forall \xi \in \mathbb{R}^{N} .
$$

Recall that the polar function $\phi^{0}: \Omega \times \mathbb{R}^{N} \rightarrow \mathbb{R}$ of $\phi$ defined by

$$
\phi^{0}\left(x, \xi^{*}\right)=\sup \left\{\left\langle\xi^{*}, \xi\right\rangle: \xi \in \mathbb{R}^{N}, \phi(x, \xi) \leq 1\right\} .
$$

Let

$$
\mathcal{K}_{\phi}(\Omega):=\left\{\mathbf{z} \in X_{\infty}(\Omega): \phi^{0}(x, \mathbf{z}(x)) \leq 1 \text { for a.e } x \in \Omega,[\mathbf{z}, \nu]=0\right\} .
$$

Definition 6.1 ([24]). Let $u \in L^{1}(\Omega)$. We define the $\phi$-total variation of $u$ in $\Omega$ as

We set

$$
\int_{\Omega}|D u|_{\phi}:=\sup \left\{\int_{\Omega} u \operatorname{div} \mathbf{z} d x: \mathbf{z} \in \mathcal{K}_{\phi}(\Omega)\right\} .
$$

$$
B V_{\phi}(\Omega):=\left\{u \in L^{1}(\Omega): \int_{\Omega}|D u|_{\phi}<\infty\right\} .
$$

From the definition it follows that $u \in L^{1}(\Omega) \rightarrow \int_{\Omega}|D u|_{\phi}$ is a lower-semicontinuous functional with respect to the $L^{1}$-convergence.

It is easy to see that if $u \in B V(\Omega)$, then

$$
\int_{\Omega}|D u|_{\phi} \leq \Gamma \int_{\Omega}|D u|
$$

Moreover, if $\phi$ is coercive in $\Omega$, that is, there exist $\lambda>0$ such that $\lambda\|\xi\| \leq \phi(x, \xi)$ for all $x \in \Omega$ and for every $\xi \in \mathbb{R}^{N}$, and continuous in second variable, in [1] it is proved that $B V_{\phi}(\Omega)=B V(\Omega)$ and

$$
\lambda \int_{\Omega}|D u| \leq \int_{\Omega}|D u|_{\phi} \leq \Gamma \int_{\Omega}|D u|
$$

In [14] (see also [24]) the following result is proved.

Proposition 6.2. Given a metric integrand $\phi$, let

$$
J_{\phi}(u):= \begin{cases}\int_{\Omega} \phi(x, \nabla u(x)) d x & \text { if } \quad u \in W^{1,1}(\Omega), \\ +\infty & \text { if } \quad u \in L^{1}(\Omega) \backslash W^{1,1}(\Omega) .\end{cases}
$$

Let $\overline{J_{\phi}}$ be the relaxed functional, that is,

$$
\overline{J_{\phi}}(u):=\inf \left\{\liminf _{n \rightarrow \infty} J_{\phi}\left(u_{n}\right): u_{n} \rightarrow u \text { in } L^{1}(\Omega), u_{n} \in W^{1,1}(\Omega)\right\} .
$$

Then, for every $u \in B V_{\phi}(\Omega)$, we have

$$
\overline{J_{\phi}}(u)=\int_{\Omega}|D u|_{\phi}
$$

Hence, for every $u \in B V_{\phi}(\Omega)$, there exists a sequence $u_{n} \in W^{1,1}(\Omega)$ such that $u_{n} \rightarrow u$ in $L^{1}(\Omega)$ and

In particular, $B V_{\phi}(\Omega)$ is the finiteness domain of $\overline{J_{\phi}}$.

$$
\int_{\Omega} \phi\left(x, \nabla u_{n}(x)\right) d x \rightarrow \int_{\Omega}|D u|_{\phi}
$$


Moreover, if $u \in B V_{\phi}(\Omega) \cap L^{q}(\Omega) \quad(1 \leq q<\infty)$, then we can find a sequence $u_{n} \in W^{1,1}(\Omega) \cap$ $L^{q}(\Omega)$ such that $u_{n} \rightarrow u$ in $L^{q}(\Omega)$.

In [24], the generalized Green's formula of Anzellotti (6.2) (see [10]) is extended to the case in which the function belongs to $B V_{\phi}(\Omega)$. Given $u \in B V_{\phi}(\Omega) \cap L^{p^{\prime}}(\Omega)$ and $\mathbf{z} \in X_{p}(\Omega)$, we define the functional $(\mathbf{z}, D u): \mathcal{D}(\Omega) \rightarrow \mathbb{R}$ as

$$
\langle(\mathbf{z}, D u), \varphi\rangle:=-\int_{\Omega} u \varphi \operatorname{div}(\mathbf{z}) d x-\int_{\Omega} u \mathbf{z} \cdot \nabla \varphi d x .
$$

For $1 \leq p \leq \infty$, we denote,

$$
\mathcal{A}_{p, \phi}(\Omega):=\left\{\mathbf{z} \in X_{p}(\Omega):\left\|\phi^{0}(x, \mathbf{z}(x))\right\|_{L^{\infty}(\Omega)}<\infty\right\} .
$$

The following result can be proved as in [10] (see also [4]).

Proposition 6.3. Assume $\phi$ is a metric integrand. If $u \in B V_{\phi}(\Omega) \cap L^{p^{\prime}}(\Omega)$ and $\mathbf{z} \in \mathcal{A}_{p, \phi}(\Omega)$, then $(\mathbf{z}, D u)$ is a Radon measure in $\Omega$ and

$$
\left|\int_{\Omega}(\mathbf{z}, D u)\right| \leq\left\|\phi_{g}^{0}(\cdot, \mathbf{z}(\cdot))\right\|_{L^{\infty}(\Omega)} \int_{\Omega}|D u|_{\phi} .
$$

Moreover, if $[\mathbf{z}, \nu]=0$ on $\partial \Omega$, the following Green's formula holds,

$$
\int_{\Omega} u \operatorname{div}(\mathbf{z}) d x+\int_{\Omega}(\mathbf{z}, D u)=0 .
$$

As a consequence of Green's formula (6.5), we have

$$
\int_{\Omega}|D u|_{\phi}:=\sup \left\{\int_{\Omega}(\mathbf{z}, D u): \mathbf{z} \in \mathcal{K}_{\phi}(\Omega)\right\} .
$$

A particular case, interesting for our purposes, is when $g: \Omega \rightarrow[0, \infty)$ is a bounded Borel function and we consider the metric integrand $\phi_{g}: \Omega \times \mathbb{R}^{N} \rightarrow[0,+\infty]$ defined by $\phi_{g}(x, \xi):=g(x)\|\xi\|$. Then (see [1])

$$
\phi_{g}^{0}\left(x, \xi^{*}\right)=\left\{\begin{array}{lll}
0 & \text { if } \quad g(x)=0, \xi^{*}=0 \\
+\infty & \text { if } \quad g(x)=0, \xi^{*} \neq 0 \\
\frac{\left\|\xi^{*}\right\|}{g(x)} & \text { if } \quad g(x)>0, \xi^{*} \in \mathbb{R}^{N}
\end{array}\right.
$$

Consequently,

$$
\mathcal{K}_{g}(\Omega):=\mathcal{K}_{\phi_{g}}(\Omega)=\left\{\mathbf{z} \in X_{\infty}(\Omega):\|\mathbf{z}(x)\| \leq g(x) \text { for a.e } x \in \Omega,[\mathbf{z}, \nu]=0\right\} .
$$

In this particular case we will use the notation

$$
B V_{g}(\Omega):=\left\{u \in L^{1}(\Omega): \int_{\Omega}|D u|_{g}<\infty\right\},
$$

where

$$
\int_{\Omega}|D u|_{g}:=\sup \left\{\int_{\Omega} u \operatorname{div} \mathbf{z} d x: \mathbf{z} \in \mathcal{K}_{g}(\Omega)\right\}=\sup \left\{\int_{\Omega}(\mathbf{z}, D u): \mathbf{z} \in \mathcal{K}_{g}(\Omega)\right\} .
$$


We define the energy functional $\Phi_{g}: L^{2}(\Omega) \rightarrow[0,+\infty]$, associated with the problem $N_{1}^{g}\left(u_{0}\right)$, by

$$
\Phi_{g}(u):=\left\{\begin{array}{lll}
\int_{\Omega}|D u|_{g} & \text { if } & u \in B V_{g}(\Omega) \cap L^{2}(\Omega) \\
+\infty & \text { if } & u \in L^{2}(\Omega) \backslash B V_{g}(\Omega) .
\end{array}\right.
$$

We have that $\Phi_{g}$ is convex and lower semi-continuous. Therefore, the subdifferential $\partial \Phi_{g}$ of $\Phi_{g}$, i.e. the operator in $L^{2}(\Omega)$ defined by

$$
v \in \partial \Phi_{g}(u) \Longleftrightarrow \Phi_{g}(w)-\Phi_{g}(u) \geq \int_{\Omega} v(w-u) d x, \quad \forall w \in L^{2}(\Omega)
$$

is a maximal monotone operator in $L^{2}(\Omega)$. Consequently, the existence and uniqueness of a solution of the abstract Cauchy problem

$$
\begin{cases}u^{\prime}(t)+\partial \Phi_{g}(u(t)) \ni 0 & t \in] 0, \infty[ \\ u(0)=u_{0} & u_{0} \in L^{2}(\Omega)\end{cases}
$$

follows immediately from the Nonlinear Semigroup Theory (see [19]). Now, to get the full strength of the abstract result derived from Semigroup Theory we need to characterize $\partial \Phi_{g}$.

Lemma 6.4. The following assertions are equivalent:

(a) $(u, v) \in \partial \Phi_{g}$;

$$
u \in L^{2}(\Omega) \cap B V_{g}(\Omega), v \in L^{2}(\Omega),
$$

$$
\exists \mathbf{z} \in X(\Omega)_{2},\|\mathbf{z}(x)\| \leq g(x), \text { a.e. } x \in \Omega \text { such that } v=-\operatorname{div}(\mathbf{z}) \text { in } \mathcal{D}^{\prime}(\Omega),
$$
and

(c) (6.8) and (6.9) hold, and

$$
\int_{\Omega}(w-u) v d x \leq \int_{\Omega} \mathbf{z} \cdot \nabla w d x-\int_{\Omega}|D u|_{g}, \quad \forall w \in W^{1,1}(\Omega) \cap L^{2}(\Omega)
$$

(d) (6.8) and (6.9) hold, and

$$
\int_{\Omega}(w-u) v d x \leq \int_{\Omega}(\mathbf{z}, D w)-\int_{\Omega}|D u|_{g} \quad \forall w \in L^{2}(\Omega) \cap B V_{g}(\Omega) ;
$$

Proof. First let us see the equivalence of (a) and (b). This follows working as in the proof of Proposition 1.10 in [4]. If we denote by

$$
\widetilde{\Phi_{g}}(v):=\sup \left\{\frac{\int_{\Omega} w v d x}{\Phi_{g}(w)}: w \in L^{2}(\Omega)\right\},
$$

since $\Phi_{g}$ is positive homogeneous of degree 1 , by Theorem 1.8 in [4], we have

$$
(u, v) \in \partial \Phi_{g} \Longleftrightarrow \widetilde{\Phi_{g}}(v) \leq 1, \quad \int_{\Omega} v u d x=\Phi_{g}(u) .
$$


Let us define for $v \in L^{2}(\Omega)$

$$
\Psi_{g}(v):=\left\{\begin{array}{lll}
\inf \left\{\left\|\phi_{g}^{0}(\cdot, \mathbf{z}(\cdot))\right\|_{L^{\infty}(\Omega)}: \mathbf{z} \in \mathcal{C}(v)\right\} & \text { if } \quad \mathcal{C}(v) \neq \emptyset \\
+\infty & \text { if } \quad \mathcal{C}(v)=\emptyset
\end{array}\right.
$$

where

$$
\mathcal{C}(v):=\left\{\mathbf{z} \in \mathcal{A}_{2, \phi_{g}}(\Omega): v=-\operatorname{div}(\mathbf{z}) \text { in } \mathcal{D}^{\prime}(\Omega),[\mathbf{z}, \nu]=0 \quad \mathcal{H}^{N-1} \text { - a.e. on } \partial \Omega\right\} .
$$

We claim that

$$
\Psi_{g}=\widetilde{\Phi_{g}}
$$

Let $v \in L^{2}(\Omega)$. If $\Psi_{g}(v)=+\infty$, then $\widetilde{\Phi_{g}}(v) \leq \Psi_{g}(v)$. Then, we may assume $\Psi_{g}(v)<\infty$. Let $\mathbf{z} \in \mathcal{C}(v)$ such that $\left\|\phi_{g}^{0}(\cdot, \mathbf{z}(\cdot))\right\|_{L^{\infty}(\Omega)}<\infty$. By Proposition 6.3, for any $u \in B V_{g}(\Omega) \cap L^{2}(\Omega)$ we have

$$
\int_{\Omega} u v d x=\int_{\Omega}(\mathbf{z}, D u) \leq\left\|\phi_{g}^{0}(\cdot, \mathbf{z}(\cdot))\right\|_{L^{\infty}(\Omega)} \int_{\Omega}|D u|_{\phi} .
$$

Taking supremums in $u$ we obtain $\widetilde{\Phi_{g}}(v) \leq\left\|\phi_{g}^{0}(\cdot, \mathbf{z}(\cdot))\right\|_{L^{\infty}(\Omega)}$. Now, taking infimums in $\mathbf{z}$ we obtain $\widetilde{\Phi_{g}}(v) \leq \Psi_{g}(v)$. To prove the opposite inequality, let $\mathcal{D}:=\left\{-\operatorname{div}(\mathbf{z}): \mathbf{z} \in \mathcal{C}(v), v \in L^{2}(\Omega)\right\}$. Then, for $u \in B V_{g}(\Omega) \cap L^{2}(\Omega)$, we have

$$
\begin{gathered}
\widetilde{\Psi_{g}}(u):=\sup \left\{\frac{\int_{\Omega} u w d x}{\Psi_{g}(w)}: w \in L^{2}(\Omega)\right\} \geq \sup \left\{\frac{\int_{\Omega} u w d x}{\Psi_{g}(w)}: w \in \mathcal{D}\right\} \\
\geq \sup \left\{\frac{-\int_{\Omega} u \operatorname{div}(\mathbf{z}) d x}{\left\|\phi_{g}^{0}(\cdot, \mathbf{z}(\cdot))\right\|_{L^{\infty}(\Omega)}}: \mathbf{z} \in \mathcal{C}(w), \quad w \in L^{2}(\Omega)\right\}=\Phi_{g}(u) .
\end{gathered}
$$

Hence, $\Psi_{g}(u) \leq \widetilde{\Phi_{g}}(u)$, and $(6.15)$ holds.

By (6.14) and (6.15), it follows the equivalence between (a) and (b). To obtain (d) from (b) is sufficient to multiply both terms of the equation $v=-\operatorname{div}(\mathbf{z})$ by $w-u$, for $w \in L^{2}(\Omega) \cap B V_{g}(\Omega)$ and to use Green's formula (6.5). It is clear that (d) implies (c). To prove that (b) follows from (d), we chose $w=u$ in (6.13) and having in mind Proposition 6.3 and (6.9), we obtain that

$$
\int_{\Omega}|D u|_{g} \leq \int_{\Omega}(\mathbf{z}, D u) \leq\left\|\phi_{g}^{0}(\cdot, \mathbf{z}(\cdot))\right\|_{L^{\infty}(\Omega)} \int_{\Omega}|D u|_{\phi} \leq \int_{\Omega}|D u|_{g},
$$

from where (6.10) follows. To obtain (6.11), we choose $w=u \pm \varphi$ in (6.13) with $\varphi \in B V(\Omega) \cap$ $C^{\infty}(\Omega) \cap W^{1,1}(\Omega)$ and we get

$$
\pm \int_{\Omega} \varphi v d x \leq \pm \int_{\Omega} \mathbf{z} \cdot \nabla \varphi d x
$$

from where it follows that

$$
\int_{\Omega} \varphi \operatorname{div}(\mathbf{z}) d x+\int_{\Omega} \mathbf{z} \cdot \nabla \varphi d x=0
$$

Then, having in mind the definition of the weak trace on $\partial \Omega$ of the normal component of $\mathbf{z}$ given in [10], we get

$$
[\mathbf{z}, \nu]=0 \quad \mathcal{H}^{N-1}-\text { a.e. on } \partial \Omega \text {. }
$$


In order to prove that (c) implies (d), let $w \in L^{2}(\Omega) \cap B V_{g}(\Omega)$. By Proposition 6.2, there exists a sequence $w_{n} \in W^{1,1}(\Omega) \cap L^{2}(\Omega)$ such that

$$
w_{n} \rightarrow w \quad \text { in } L^{2}(\Omega) \quad \text { and } \quad \int_{\Omega} g(x)\left|\nabla w_{n}(x)\right| d x \rightarrow \int_{\Omega}|D w|_{g} .
$$

Using $w_{n}$ as test function in (6.12), we have

$$
\int_{\Omega}\left(w_{n}-u\right) v d x \leq \int_{\Omega} \mathbf{z} \cdot \nabla w_{n} d x-\int_{\Omega}|D u|_{g} .
$$

Now, by Lemma 13.2 in [24], we have

$$
\int_{\Omega} \mathbf{z} \cdot \nabla w_{n} d x \rightarrow \int_{\Omega}(\mathbf{z}, D w) .
$$

Therefore, taking limit as $n \rightarrow+\infty$ in (6.16), we get (6.13).

Definition 6.5. We say that $u \in C\left([0, T] ; L^{2}(\Omega)\right)$ is a solution of problem $N_{1}^{g}\left(u_{0}\right)$ in $[0, T] \times \Omega$ if $u \in W_{l o c}^{1,2}\left(0, T ; L^{2}(\Omega)\right) u(t) \in B V_{g}(\Omega)$ for almost all $\left.t \in\right] 0, T\left[, u(0)=u_{0}\right.$, and there exists $\mathbf{z} \in L^{\infty}(] 0, T\left[\times \Omega ; \mathbb{R}^{N}\right),\|\mathbf{z}(t, x)\| \leq g(x)$, a.e. $\left.(t, x) \in\right] 0, T[\times \Omega$ such that $[\mathbf{z}(t), \nu]=0$ in $\partial \Omega$ a.e. $t \in] 0, T[$, satisfying

and

$$
u_{t}=\operatorname{div}(\mathbf{z}) \quad \text { in } \mathcal{D}^{\prime}(] 0, T[\times \Omega)
$$

$$
\int_{\Omega}(u(t)-w) u_{t} d x \leq \int_{\Omega}(\mathbf{z}, D w)-\int_{\Omega}|D u(t)|_{g}
$$

for all $w \in L^{2}(\Omega) \cap B V_{g}(\Omega)$ an a.e. $t \in[0, T]$.

By Lemma 6.4, the concept of solution for problem $N_{1}^{g}\left(u_{0}\right)$ coincides with the concept of strong solution for the abstract Cauchy problem (6.7). Then, since we know that problem (6.7) has a unique strong solution for any initial data in $L^{2}(\Omega)$, we have the following existence and uniqueness result.

Theorem 6.6. Let $g: \Omega \rightarrow[0, \infty)$ is a bounded Borel function. For any initial data $u_{0} \in L^{2}(\Omega)$ there exists a unique solution $u$ of the problem $N_{1}^{g}\left(u_{0}\right)$ in $[0, T] \times \Omega$ for every $T>0$. Moreover if $u$ and $v$ are solutions of $N_{1}^{g}\left(u_{0}\right)$ corresponding to the initial conditions $u_{0}, v_{0} \in L^{2}(\Omega)$, then

$$
\|u(t)-v(t)\|_{L^{2}(\Omega)} \leq\left\|u_{0}-v_{0}\right\|_{L^{2}(\Omega)} \quad \text { for any } t>0 .
$$

7. Convergence of the nonlocal problems to the local problem. The Case $p=1$

Similarly to the case $p>1$, in order to do the rescaling, we need a variant of $[7$, Proposition 3.2(1.ii)].

Proposition 7.1. Let $\rho: \mathbb{R}^{N} \rightarrow \mathbb{R}$ be a nonnegative continuous radial function with compact support, non-identically zero, and $\rho_{n}(x):=n^{N} \rho(n x)$. Let $S$ an open set, $S \subset \Omega$, and let $l \in$ $L^{\infty}\left(\mathbb{R}^{N}\right)$ such that

$$
l(x)= \begin{cases}l(x)>0 & \text { in } S \\ 0 & \text { in } \mathbb{R}^{N} \backslash S .\end{cases}
$$

Let us also assume that $l$ satisfies

$$
l \in C(S)
$$


Let $\left\{f_{n}\right\}$ be a sequence of functions in $L^{1}(\Omega)$ such that

$$
\int_{\Omega} \int_{\Omega} \rho_{n}(y-x) l\left(\frac{x+y}{2}\right)\left|f_{n}(y)-f_{n}(x)\right| d x d y \leq M \frac{1}{n}
$$

and $\left\{f_{n}\right\}$ is weakly convergent in $L^{1}(S)$ to $f$.

Then, $l \frac{\partial f}{\partial w_{j}}$ is a bounded Radon measure in $S, j=1, \ldots, N$, and moreover

$$
\lim _{n}\left[\rho(z) l(w) \chi_{\Omega}\left(w+\frac{1}{2 n}\right) \chi_{\Omega}\left(w-\frac{1}{2 n} z\right) \frac{\bar{f}_{n}\left(w+\frac{1}{2 n} z\right)-\bar{f}_{n}\left(w-\frac{1}{2 n} z\right)}{1 / n}\right]=\mu(w, z)
$$

weakly as measures with

$$
\mu(w, z)=\rho(z) l(w) z \cdot \nabla f(w) \quad \text { in } S \times \mathbb{R}^{N},
$$

and

$$
\mu(w, z)=0 \quad \text { in }\left(\mathbb{R}^{N} \backslash \bar{\Omega}\right) \times \mathbb{R}^{N} .
$$

Proof. Making the change of variables $y=x+\frac{1}{n} z, x=w-\frac{1}{2 n} z$, we rewrite (7.2) as

$$
\int_{\mathbb{R}^{N}} \int_{\mathbb{R}^{N}} \rho(z) l(w) \chi_{\Omega}^{\times}\left(w \pm \frac{1}{2 n} z\right)\left|\frac{\bar{f}_{n}\left(w+\frac{1}{2 n} z\right)-\bar{f}_{n}\left(w-\frac{1}{2 n} z\right)}{1 / n}\right| d w d z \leq M,
$$

where $\chi_{\Omega}^{\times}\left(w \pm \frac{1}{2 n} z\right)=\chi_{\Omega}\left(w+\frac{1}{2 n} z\right) \chi_{\Omega}\left(w-\frac{1}{2 n} z\right)$. Therefore, up to a subsequence,

$$
\rho(z) l(w) \chi_{\Omega}^{\times}\left(w \pm \frac{1}{2 n} z\right) \frac{\bar{f}_{n}\left(w+\frac{1}{2 n} z\right)-\bar{f}_{n}\left(w-\frac{1}{2 n} z\right)}{1 / n} \rightarrow \mu(w, z)
$$

as measures and

$$
\mu(w, z)=0 \quad \text { in }\left(\mathbb{R}^{N} \backslash \bar{\Omega}\right) \times \mathbb{R}^{N} .
$$

If $\varphi \in C_{c}^{\infty}(\Omega), \operatorname{supp}(\varphi) \subset S$, taking

$$
\hat{\varphi}= \begin{cases}\frac{\varphi}{l} & \text { in } S \\ 0 & \text { otherwise }\end{cases}
$$

and $\psi \in C_{c}^{\infty}\left(\mathbb{R}^{N}\right)$, by (7.3) and [2, Proposition 1.62], we have

$$
\begin{aligned}
\int_{\mathbb{R}^{N}} \int_{\Omega} \rho(z) l(w) \chi_{\Omega}^{\times}(w & \left. \pm \frac{1}{2 n} z\right) \frac{\bar{f}_{n}\left(w+\frac{1}{2 n} z\right)-\bar{f}_{n}\left(w-\frac{1}{2 n} z\right)}{1 / n} \hat{\varphi}(w) d w \psi(z) d z \\
& \rightarrow \int_{\mathbb{R}^{N}} \int_{\Omega} \hat{\varphi}(w) \psi(z) d \mu(w, z),
\end{aligned}
$$

That is,

$$
\begin{aligned}
\int_{\mathbb{R}^{N}} \int_{S} \rho(z) \chi_{\Omega}^{\times}(w & \left. \pm \frac{1}{2 n} z\right) \frac{\bar{f}_{n}\left(w+\frac{1}{2 n} z\right)-\bar{f}_{n}\left(w-\frac{1}{2 n} z\right)}{1 / n} \varphi(w) d w \psi(z) d z \\
& \rightarrow \int_{\mathbb{R}^{N}} \int_{S} \frac{1}{l(w)} \varphi(w) \psi(z) d \mu(w, z) .
\end{aligned}
$$


Now, for $n$ large enough,

$$
\begin{aligned}
\int_{\mathbb{R}^{N}} & \int_{S} \rho(z) \chi_{\Omega}^{\times}\left(w \pm \frac{1}{2 n} z\right) \frac{\bar{f}_{n}\left(w+\frac{1}{2 n} z\right)-\bar{f}_{n}\left(w-\frac{1}{2 n} z\right)}{1 / n} \varphi(w) d w \psi(z) d z \\
& =\int_{\mathbb{R}^{N}} \rho(z) \int_{S} \frac{\bar{f}_{n}\left(w+\frac{1}{2 n} z\right)-\bar{f}_{n}\left(w-\frac{1}{2 n} z\right)}{1 / n} \varphi(w) d w \psi(z) d z \\
& =-\int_{\mathbb{R}^{N}} \rho(z) \int_{S} f_{n}(w) \frac{\bar{\varphi}\left(w+\frac{1}{2 n} z\right)-\bar{\varphi}\left(w-\frac{1}{2 n} z\right)}{1 / n} d w \psi(z) d z .
\end{aligned}
$$

Then, passing to the limit, on account of (7.4), we get

$$
\int_{\mathbb{R}^{N}} \int_{S} \frac{1}{l(w)} \varphi(w) \psi(z) d \mu(w, z)=-\int_{\mathbb{R}^{N}} \int_{S} \rho(z) f(w) z \cdot \nabla \varphi(w) \psi(z) d w d z .
$$

Now, applying the disintegration theorem (Theorem 2.28 in [2]) to the measure $\mu$, we get that if $\pi: S \times \mathbb{R}^{N} \rightarrow \mathbb{R}^{N}$ is the projection on the first factor and $\nu=\pi_{\#}|\mu|$, then there exists a Radon measures $\mu_{w}$ in $\mathbb{R}^{N}$ such that $w \mapsto \mu_{w}$ is $\nu$-measurable,

$$
\left|\mu_{w}\right|\left(\mathbb{R}^{N}\right) \leq 1 \quad \nu \text { - a.e. } \quad \text { in } S
$$

and, for any $h \in L^{1}\left(S \times \mathbb{R}^{N},|\mu|\right)$,

$$
h(w, \cdot) \in L^{1}\left(\mathbb{R}^{N},\left|\mu_{w}\right|\right) \quad \nu \text { - a.e. } \quad \text { in } w \in S, \quad w \mapsto \int_{\mathbb{R}^{N}} h(w, z) d \mu_{w}(z) \in L^{1}(S, \nu)
$$

and

$$
\int_{S \times \mathbb{R}^{N}} h(w, z) d \mu(w, z)=\int_{S}\left(\int_{\mathbb{R}^{N}} h(w, z) d \mu_{w}(z)\right) d \nu(w) .
$$

From (7.5) and (7.6), we get, for $\varphi \in C_{c}^{\infty}(S)$ and $\psi \in C_{c}^{\infty}\left(\mathbb{R}^{N}\right)$,

$$
\int_{S}\left(\int_{\mathbb{R}^{N}} \psi(z) d \mu_{w}(z)\right) \frac{1}{l(w)} \varphi(w) d \nu(w)=\left\langle\sum_{i=1}^{N} \int_{\mathbb{R}^{N}} \rho(z) z_{i} \psi(z) d z \frac{\partial f}{\partial w_{i}}, \varphi\right\rangle .
$$

Hence, as measures,

$$
\sum_{i=1}^{N} \int_{\mathbb{R}^{N}} \rho(z) z_{i} \psi(z) d z \frac{\partial f}{\partial w_{i}}=\int_{\mathbb{R}^{N}} \psi(z) d \mu_{w}(z) \frac{1}{l} \nu \quad \text { in } S
$$

and therefore

$$
\sum_{i=1}^{N} \int_{\mathbb{R}^{N}} \rho(z) z_{i} \psi(z) d z l \frac{\partial f}{\partial w_{i}}=\int_{\mathbb{R}^{N}} \psi(z) d \mu_{w}(z) \nu \quad \text { in } S .
$$

Let now $\tilde{\psi} \in C_{c}^{\infty}\left(\mathbb{R}^{N}\right)$ be a radial function such that $\tilde{\psi}=1$ in $\operatorname{supp}(\rho)$. Taking $\psi(z)=\tilde{\psi}(z) z_{j}$ in the above expression and having in mind that

$$
\int_{\mathbb{R}^{N}} \rho(z) z_{i} z_{j} \tilde{\psi}(z) d z=0 \quad \text { if } i \neq j
$$

we get

$$
\int_{\mathbb{R}^{N}} \rho(z) z_{j}^{2} d z l \frac{\partial f}{\partial w_{j}}=\int_{\mathbb{R}^{N}} \tilde{\psi}(z) z_{j} d \mu_{w}(z) \nu \quad \text { in } S .
$$


Since $\nu \in M_{b}(S)$ and $w \mapsto \int_{\mathbb{R}^{N}} \tilde{\psi}(z) z_{j} d \mu_{w}(z) \in L^{1}(S, \nu)$, we obtain that $l \frac{\partial f}{\partial w_{j}}$ is a bounded Radon measure in $S$. Going back to (7.6), we get

$$
\mu(w, z)=l(w) \sum_{i=1}^{N} \frac{\partial f}{\partial w_{i}}(x) \cdot \rho(z) z_{i} \mathcal{L}^{N}(z) .
$$

For the proof our next results we need the following assumptions: we assume that $g \in L^{\infty}\left(\mathbb{R}^{N}\right)$ is such that

$$
g(x)= \begin{cases}g(x)>0 & \text { a.e. in } S \subset \Omega, S \text { an open set, } \\ 0 & \text { a.e. in } \mathbb{R}^{N} \backslash S, \\ & g \in C(S) .\end{cases}
$$

Let us now proceed with the rescaling. Set

$$
J_{1, \varepsilon}(x):=\frac{C_{J, 1}}{\varepsilon^{1+N}} J\left(\frac{x}{\varepsilon}\right), \quad \text { with } \quad \frac{1}{C_{J, 1}}:=\frac{1}{2} \int_{\mathbb{R}^{N}} J(z)\left|z_{N}\right| d z .
$$

Theorem 7.2. Assume $J$ satisfies (HJ) and $J(x) \geq J(y)$ if $|x| \leq|y|$ and that $g$ satisfies (7.7) and (7.8). For any $\phi \in L^{\infty}(\Omega)$, we have

$$
\left(I+B_{1}^{J_{1, \varepsilon}, g}\right)^{-1} \phi \rightarrow\left(I+\partial \Phi_{g}\right)^{-1} \phi \quad \text { in } L^{1}(\Omega) \text { as } \varepsilon \rightarrow 0 .
$$

Proof. Given $\varepsilon>0$, we set $u_{\varepsilon}=\left(I+B_{1}^{J_{1, \varepsilon}, g}\right)^{-1} \phi$. Then, there exists $h_{\epsilon} \in L^{\infty}(\Omega \times \Omega), h_{\varepsilon}(x, y)=$ $-h_{\varepsilon}(y, x)$ for almost all $x, y \in \Omega,\left\|h_{\varepsilon}\right\|_{\infty} \leq 1$,

$$
J\left(\frac{x-y}{\varepsilon}\right) g\left(\frac{x+y}{2}\right) h_{\varepsilon}(x, y) \in J\left(\frac{x-y}{\varepsilon}\right) g\left(\frac{x+y}{2}\right) \operatorname{sign}\left(u_{\varepsilon}(y)-u_{\varepsilon}(x)\right) \quad \text { a.e. } x, y \in \Omega
$$

and

$$
-\frac{C_{J, 1}}{\varepsilon^{1+N}} \int_{\Omega} J\left(\frac{x-y}{\varepsilon}\right) g\left(\frac{x+y}{2}\right) h_{\varepsilon}(x, y) d y=\phi(x)-u_{\varepsilon}(x) \quad \text { a.e. } x \in \Omega .
$$

Observe that

$$
\begin{aligned}
& -\frac{C_{J, 1}}{\varepsilon^{1+N}} \int_{\Omega} \int_{\Omega} J\left(\frac{x-y}{\varepsilon}\right) g\left(\frac{x+y}{2}\right) h_{\varepsilon}(x, y) d y u_{\varepsilon}(x) d x \\
& \quad=\frac{C_{J, 1}}{\varepsilon^{1+N}} \frac{1}{2} \int_{\Omega} \int_{\Omega} J\left(\frac{x-y}{\varepsilon}\right) g\left(\frac{x+y}{2}\right)\left|u_{\varepsilon}(y)-u_{\varepsilon}(x)\right| d y d x .
\end{aligned}
$$

By (7.9), we can write

$$
\begin{array}{ll} 
& \frac{C_{J, 1}}{2 \varepsilon^{1+N}} \int_{\Omega} \int_{\Omega} J\left(\frac{x-y}{\varepsilon}\right) g\left(\frac{x+y}{2}\right) h_{\varepsilon}(x, y)(v(y)-v(x)) d x d y \\
=-\frac{C_{J, 1}}{\varepsilon^{1+N}} \int_{\Omega} \int_{\Omega} J\left(\frac{x-y}{\varepsilon}\right) g\left(\frac{x+y}{2}\right) h_{\varepsilon}(x, y) d y v(x) d x & \\
=\int_{\Omega}\left(\phi(x)-u_{\varepsilon}(x)\right) v(x) d x, & \forall v \in L^{\infty}(\Omega) .
\end{array}
$$


Since $u_{\varepsilon} \ll \phi$, there exists a sequence $\varepsilon_{n} \rightarrow 0$ such that

$$
u_{\varepsilon_{n}} \rightarrow u \quad \text { weakly in } L^{1}(\Omega), \quad u \ll \phi .
$$

Observe that $\left\|u_{\varepsilon_{n}}\right\|_{L^{\infty}(\Omega)},\|u\|_{L^{\infty}(\Omega)} \leq\|\phi\|_{L^{\infty}(\Omega)}$. Hence taking $\varepsilon=\varepsilon_{n}$ and $v=u_{\varepsilon_{n}}$ in (7.11), changing variables and having in mind (7.10), we get

$$
\begin{aligned}
& \int_{\mathbb{R}^{N}} \int_{\mathbb{R}^{N}} \frac{C_{J, 1}}{2} J(z) g(w) \chi_{\Omega}^{\times}\left(x \pm \frac{\varepsilon_{n}}{2} z\right)\left|\frac{\bar{u}_{\varepsilon_{n}}\left(w+\frac{\varepsilon_{n}}{2} z\right)-\bar{u}_{\varepsilon_{n}}\left(w-\frac{\varepsilon_{n}}{2} z\right)}{\varepsilon_{n}}\right| d w d z \\
& =\int_{\Omega} \int_{\Omega} \frac{1}{2} \frac{C_{J, 1}}{\varepsilon_{n}{ }^{N}} J\left(\frac{x-y}{\varepsilon_{n}}\right) g\left(\frac{x+y}{2}\right)\left|\frac{u_{\varepsilon_{n}}(y)-u_{\varepsilon_{n}}(x)}{\varepsilon_{n}}\right| d x d y \\
& =\int_{\Omega}\left(\phi(x)-u_{\varepsilon_{n}}(x)\right) u_{\varepsilon_{n}}(x) d x \leq M \quad \forall n \in \mathbb{N} .
\end{aligned}
$$

Therefore, by Proposition 7.1, $g \frac{\partial u}{\partial w_{j}}$ is a bounded Radon measure in $S, j=1, \ldots, N$,

$$
\frac{C_{J, 1}}{2} J(z) g(w) \chi_{\Omega}^{\times}\left(w \pm \frac{\varepsilon_{n}}{2} z\right) \frac{\bar{u}_{\varepsilon_{n}}\left(w+\frac{\varepsilon_{n}}{2} z\right)-\bar{u}_{\varepsilon_{n}}\left(w-\frac{\varepsilon_{n}}{2} z\right)}{\varepsilon_{n}} \rightarrow \mu(w, z)
$$

weakly as measures with

$$
\mu(w, z)=\frac{C_{J, 1}}{2} J(z) g(w) z \cdot D u(w) \quad \text { in } S \times \mathbb{R}^{N},
$$

and

$$
\mu(w, z)=0 \quad \text { in }\left(\mathbb{R}^{N} \backslash \bar{\Omega}\right) \times \mathbb{R}^{N} .
$$

And by [7, Proposition 3.2(2.ii)] (see also [17, Theorem 4])

$$
u_{\varepsilon_{n}} \rightarrow u \quad \text { strongly in } L^{1}(\Omega) \text {. }
$$

Moreover, we can also assume that

$$
J(z) \chi_{\Omega}^{\times}\left(w \pm \frac{\varepsilon_{n}}{2} z\right) g(w) \bar{h}_{\varepsilon_{n}}\left(w-\frac{\varepsilon_{n}}{2} z, w+\frac{\varepsilon_{n}}{2} z\right) \rightarrow \Lambda(w, z)
$$

weakly* in $L^{\infty}\left(\mathbb{R}^{N}\right) \times L^{\infty}\left(\mathbb{R}^{N}\right)$, and $|\Lambda(w, z)| \leq g(w) J(z)$ almost everywhere in $\mathbb{R}^{N} \times \mathbb{R}^{N}$. Changing variables and having in mind (7.11), we can write

$$
\begin{array}{lr}
\frac{C_{J, 1}}{2} \int_{\mathbb{R}^{N}} \int_{\mathbb{R}^{N}} J(z) \chi_{\Omega}^{\times}\left(w \pm \frac{\varepsilon_{n}}{2} z\right) g(w) \bar{h}_{\varepsilon_{n}}\left(w-\frac{\varepsilon}{2} z, w+\frac{\varepsilon_{n}}{2} z\right) \frac{\bar{v}\left(w+\frac{\varepsilon_{n}}{2} z\right)-\bar{v}\left(w-\frac{\varepsilon_{n}}{2} z\right)}{\varepsilon_{n}} d z d w \\
=\int_{\Omega}\left(\phi(x)-u_{\varepsilon_{n}}(x)\right) v(x) d x & \forall v \in L^{\infty}(\Omega) .
\end{array}
$$

By (7.13), passing to the limit in (7.14), we get

$$
\frac{C_{J, 1}}{2} \int_{\mathbb{R}^{N}} \int_{S} \Lambda(w, z) z \cdot \nabla v(w) d w d z=\int_{\Omega}(\phi-u) v \quad \forall v \text { smooth, }
$$

and, by approximation, $\forall v \in L^{\infty}(\Omega) \cap W^{1,1}(\Omega)$. We set $\zeta=\left(\zeta_{1}, \ldots, \zeta_{N}\right)$, the vector field defined by

$$
\zeta_{j}(w):=\frac{C_{J, 1}}{2} \int_{\mathbb{R}^{N}} \Lambda(w, z) z_{j} d z, \quad j=1, \ldots, N .
$$


Then, $\zeta \in L^{\infty}\left(\Omega, \mathbb{R}^{N}\right)$, and from $(7.15)$,

$$
-\operatorname{div}(\zeta)=\phi-u \quad \text { in } \mathcal{D}^{\prime}(\Omega)
$$

Given $\xi \in \mathbb{R}^{N} \backslash\{0\}$, let $R_{\xi}$ be the rotation such that $R_{\xi}^{t}(\xi)=\mathbf{e}_{1}|\xi|$. If we make the change of variables $z=R_{\xi}(y)$, we obtain

$$
\begin{aligned}
\zeta(x) \cdot \xi & =\frac{C_{J, 1}}{2} \int_{\mathbb{R}^{N}} \Lambda(x, z) z \cdot \xi d z=\frac{C_{J, 1}}{2} \int_{\mathbb{R}^{N}} \Lambda\left(x, R_{\xi}(y)\right) R_{\xi}(y) \cdot \xi d y \\
& =\frac{C_{J, 1}}{2} \int_{\mathbb{R}^{N}} \Lambda\left(x, R_{\xi}(y)\right) y_{1}|\xi| d y .
\end{aligned}
$$

On the other hand, since $J$ is a radial function and $\Lambda(w, z) \leq g(w) J(z)$ almost everywhere, we obtain, $C_{J, 1}{ }^{-1}=\frac{1}{2} \int_{\mathbb{R}^{N}} J(z)\left|z_{1}\right| d z$ and

$$
|\zeta(w) \cdot \xi| \leq \frac{C_{J, 1}}{2} \int_{\mathbb{R}^{N}} g(w) J(y)\left|y_{1}\right| d y|\xi|=g(w)|\xi| \quad \text { a.e. } w \in \mathbb{R}^{N} .
$$

Therefore, $\|\zeta(w)\|_{l^{2}(N)} \leq g(w)$ a.e. $w \in \mathbb{R}^{N}$.

Since $u \in L^{\infty}(\Omega), u \in B V_{g}(\Omega)$ and $\int_{\Omega}|D u|_{g} \leq|g D u|(S)$, by Lemma 6.4, to finish the proof we only need to show that

$$
\int_{\Omega}(\rho-u)(\phi-u) \leq \int_{S} \zeta \cdot \nabla \rho-|g D u|(S) \quad \forall \rho \in W^{1,1}(\Omega) .
$$

Given $\rho \in W^{1,1}(\Omega)$, taking $v=\rho-u_{\varepsilon_{n}}$ in (7.14), we get

$$
\begin{aligned}
& \int_{\Omega}\left(\phi(x)-u_{\varepsilon_{n}}(x)\right)\left(\rho(x)-u_{\varepsilon_{n}}(x)\right) d x \\
& =\frac{C_{J, 1}}{2} \int_{\mathbb{R}^{N}} \int_{\mathbb{R}^{N}} J(z) g(w) \chi_{\Omega}^{\times}\left(w \pm \frac{\varepsilon_{n}}{2} z\right) \bar{h}_{\varepsilon_{n}}\left(w-\frac{\varepsilon_{n}}{2} z, w+\frac{\varepsilon_{n}}{2} z\right) \times \\
& \quad \times\left(\frac{\bar{\rho}\left(w+\frac{\varepsilon_{n}}{2} z\right)-\rho\left(w-\frac{\varepsilon_{n}}{2} z\right)}{\varepsilon_{n}}-\frac{\bar{u}_{\varepsilon_{n}}\left(w+\frac{\varepsilon_{n}}{2} z\right)-\bar{u}_{\varepsilon_{n}}\left(w-\frac{\varepsilon_{n}}{2} z\right)}{\varepsilon_{n}}\right) d z d w .
\end{aligned}
$$

Having in mind (7.12) and (7.13) and taking limit in (7.17) as $n \rightarrow \infty$, we obtain that

$$
\begin{aligned}
\int_{\Omega}(\rho-u)(\phi-u) d x \leq & \frac{C_{J, 1}}{2} \int_{S} \int_{\mathbb{R}^{N}} \Lambda(w, z) z \cdot \nabla \rho(w) d z d w-\frac{C_{J, 1}}{2} \int_{S} \int_{\mathbb{R}^{N}}|g(w) J(z) z \cdot D u| d z d w \\
& =\int_{S} \zeta \cdot \nabla \rho-\frac{C_{J, 1}}{2} \int_{S} \int_{\mathbb{R}^{N}}|g(w) J(z) z \cdot D u| d z d w .
\end{aligned}
$$

Now, for every $w \in S$ such that the Radon-Nikodym derivative $\frac{g D u}{|g D u|}(w) \neq 0$, let $R_{w}$ be the rotation such that $R_{w}^{t}\left(\frac{g D u}{|g D u|}(w)\right)=\mathbf{e}_{1}\left|\frac{g D u}{|g D u|}(w)\right|$. Then, since $J$ is a radial function and $\left|\frac{g D u}{|g D u|}(w)\right|=1|g D u|$-a.e. in $S$, if we make the change of variables $y=R_{w}(z)$, we have

$$
\begin{gathered}
\frac{C_{J, 1}}{2} \int_{S} \int_{\mathbb{R}^{N}}|g(w) J(z) z \cdot D u| d z d w=\frac{C_{J, 1}}{2} \int_{S} \int_{\mathbb{R}^{N}} J(z)\left|z \cdot \frac{g D u}{|g D u|}(w)\right| d z d|g D u|(w) \\
=\frac{C_{J, 1}}{2} \int_{S} \int_{\mathbb{R}^{N}} J(y)\left|y_{1}\right| d y d|g D u|(w)=\int_{S}|g D u| .
\end{gathered}
$$

Therefore (7.16) holds. 
As a consequence of Theorem 7.2 and the Nonlinear Semigroup Theory (see [20]), we have the following convergence result.

Theorem 7.3. Assume $J$ satisfies (HJ) and $J(x) \geq J(y)$ if $|x| \leq|y|$, and $g$ satisfies (7.7) and (7.8). Let $T>0$ and $u_{0} \in L^{2}(\Omega)$. Let $u_{\varepsilon}$ the unique solution of $P_{1}^{J_{1, \varepsilon}, g}\left(u_{0}\right)$ and $u$ the unique solution of $N_{1}^{g}\left(u_{0}\right)$. Then

$$
\lim _{\varepsilon \rightarrow 0} \sup _{t \in[0, T]}\left\|u_{\varepsilon}(t, \cdot)-u(t, \cdot)\right\|_{L^{1}(\Omega)}=0 .
$$

Acknowledgements. Part of this work was performed during a visit of JDR to Univ. de Valencia. He is grateful for the warm hospitality and the stimulating working atmosphere found there. FA, JMM and JT are partially supported by the Spanish MCI and FEDER, project MTM2008-03176. JDR is partially supported by ANPCyT PICT 5009, UBA X066, Fundación Antorchas and CONICET (Argentina).

\section{REFERENCES}

[1] M. Amar, and G. Bellettini, A notion of total variation dependding on a metric with discontinuous coeficients, Ann. Inst. Henri Poincaré Analyse non linéaire 11 (1994), 91-133.

[2] L. Ambrosio, N. Fusco and D. Pallara, Functions of Bounded Variation and Free Discontinuity Problems, Oxford Mathematical Monographs, 2000.

[3] F. Andreu, C. Ballester, V. Caselles and J. M. Mazon, Minimizing Total Variation Flow, Diff. and Int. Eq. 14 (2001), 321-360.

[4] F. Andreu, V. Caselles, and J.M. Mazon, Parabolic Quasilinear Equations Minimizing Linear Growth Functionals, Progress in Mathematics, vol. 223, 2004. Birkhauser.

[5] F. Andreu, J.M. Mazon, S. Segura and J. Toledo, Quasilinear Elliptic and Parabolic Equations in $L^{1}$ with Nonlinear Boundary Conditions, Adv. in Math. Sci. and Appl. 7 (1997), 183-213.

[6] F. Andreu, J. M. Mazón, J. D. Rossi and J. Toledo. The Neumann problem for nonlocal nonlinear diffusion equations. J. Evol. Equations 8 (2008), 189-215.

[7] F. Andreu, J. M. Mazón, J. D. Rossi and J. Toledo. A nonlocal p-laplacian evolution equation with neumann boundary conditions. J. Math. Pures Appl. 90 (2008), 201-227.

[8] F. Andreu, J. M. Mazón, J. D. Rossi and J. Toledo. The limit as $p \rightarrow \infty$ in a nonlocal p-Laplacian evolution equation. A nonlocal approximation of a model for sandpiles. To appear in Calc. Var. PDE. Doi:10.1007/s00526-008-0205-2.

[9] F. Andreu, J. M. Mazón, J. D. Rossi and J. Toledo. A nonlocal p-Lapla-cian evolution equation with non homogeneous Dirichlet boundary conditions. SIAM J. Math. Anal. 40 (2009), 1815-1851.

[10] G. Anzellotti, Pairings Between Measures and Bounded Functions and Compensated Compactness, Ann. di Matematica Pura ed Appl. IV (135) (1983), 293-318.

[11] P. Bates and A. Chmaj. An integrodifferential model for phase transitions: stationary solutions in higher dimensions. J. Statistical Phys. 95 (1999), 1119-1139.

[12] P. Bates and A. Chmaj. A discrete convolution model for phase transitions. Arch. Rat. Mech. Anal. 150 (1999), 281-305.

[13] P. Bates, P. Fife, X. Ren and X. Wang. Travelling waves in a convolution model for phase transitions. Arch. Rat. Mech. Anal. 138 (1997), 105-136.

[14] G. Bellettini, G. Bouchitté and I. Fragalá. BV functions with repect to a measure and relaxation of metric integral functionals. J. Convex Anal. 6 (1999), 349-366.

[15] Ph. Bénilan and M. G. Crandall. Completely accretive operators. In Semigroup theory and evolution equations (Delft, 1989), volume 135 of Lecture Notes in Pure and Appl. Math., pages 41-75. Dekker, New York, 1991.

[16] Ph. Bénilan, M. G. Crandall and A. Pazy. Evolution Equations Governed by Accretive Operators. Book to appear. 
[17] J. Bourgain, H. Brezis and P. Mironescu. Another look at Sobolev spaces. In: Menaldi, J. L. et al. (eds.) Optimal control and partial differential equations. A volume in honour of A. Bensoussan's 60th birthday, pages 439-455, IOS Press, 2001.

[18] H. Brezis. Équations et inéquations non linéaires dans les espaces vectoriels en dualité. Ann. Inst. Fourier 18 (1968), 115-175.

[19] H. Brezis, Operateurs Maximaux Monotones, North Holland, Amsterdam, 1973.

[20] H. Brezis and A. Pazy. Convergence and approximation of semigroups of nonlinear operators in Banach spaces. J. Funct. Anal. 9 (1972), 63-74.

[21] L. Caffarelli and L. Silvestre. An extension problem related to the fractional Laplacian. Comm. Partial Differential Equations 32(7-9) (2007), 1245-1260.

[22] L. Caffarelli, S. Salsa and L. Silvestre. Regularity estimates for the solution and the free boundary of the obstacle problem for the fractional Laplacian. Invent. Math. 171(2) (2008), 425-461.

[23] C. Carrillo and P. Fife. Spatial effects in discrete generation population models. J. Math. Biol. 50(2) (2005), $161-188$.

[24] V. Caselles, G. Facciolo and E. Meinhardt. Anisotropic Cheeger sets and applications. Preprint.

[25] V. Caselles, R. Kimmel and G. Sapiro, Geodesic Active Contours, Proc. Int.. Conf. Computer Vision, Boston, June 1995.

[26] V. Caselles, R. Kimmel and G. Sapiro, Geodesic Active Contours, Inter. Journal Computer Vision 22(1) (1997), 61-79.

[27] Y. Chen and T. Wunderli, Adaptive total variation for image restoration in BV space, J. Math. Anal. Appl. 272, (2002) 117-137.

[28] E. Chasseigne, M. Chaves and J. D. Rossi. Asymptotic behaviour for nonlocal diffusion equations. J. Math. Pures Appl. 86 (2006), 271-291.

[29] X. Chen. Existence, uniqueness and asymptotic stability of travelling waves in nonlocal evolution equations. Adv. Differential Equations 2 (1997), 125-160.

[30] S.-K. Chua. Existence theorems on weighted Sobolev spaces. Indiana Math. J. 41 (1992), 1027-1076.

[31] C. Cortázar, J. Coville, M. Elgueta and S. Martínez. A nonlocal inhomogeneous dispersal process. J. Differential Equations 241, (2007) 332-358.

[32] C. Cortazar, M. Elgueta and J. D. Rossi. A non-local diffusion equation whose solutions develop a free boundary. Annales Henri Poincaré 6 (2005), 269-281.

[33] C. Cortazar, M. Elgueta, J. D. Rossi and N. Wolanski. Boundary fluxes for non-local diffusion. J. Differential Equations 234 (2007), 360-390.

[34] C. Cortazar, M. Elgueta, J. D. Rossi and N. Wolanski. How to approximate the heat equation with Neumann boundary conditions by nonlocal diffusion problems. Arch. Rat. Mech. Anal. 187 (2008), 137-156.

[35] J. Coville, J. Davila and S. Martinez. Nonlocal anisotropic dispersal with monostable nonlinearity. J. Differential Equations 244 (2008), 3080-3118.

[36] M. G. Crandall. Nonlinear Semigroups and Evolution Governed by Accretive Operators. In Proc. of Sympos. in Pure Mathematics, Part I, Vol. 45 ( F. Browder ed.). A.M.S., Providence 1986, pages 305-338.

[37] P. Drábek, A. Kufner and F. Nicolosi. Quasilinear elliptic equations with degenerations and singularities. de Gruyter Series in Nonlinear Analysis and Applications, 5. Walter de Gruyter Co., Berlin, 1997.

[38] L. C. Evans and R. F. Gariepy, Measure Theory and Fine Properties of Functions, Studies in Advanced Math., CRC Press, 1992.

[39] P. Fife. Some nonclassical trends in parabolic and parabolic-like evolutions. Trends in nonlinear analysis, 153-191, Springer, Berlin, 2003.

[40] P. Fife and X. Wang. A convolution model for interfacial motion: the generation and propagation of internal layers in higher space dimensions. Adv. Differential Equations 3 (1998), 85-110.

[41] J. Heinonen, T. Kilpeläinen and O. Martio. Nonlinear potential theory of degenerate elliptic equations. Oxford Mathematical Monographs. Oxford Science Publications. The Clarendon Press, Oxford University Press, New York, 1993.

[42] T. Kilpeläinen, Weighted Sobolev spaces and capacity. Ann. Acad. Sci. Fenn. Ser. A I Math. 19 (1994), 95-113.

[43] L. Rudin, S. Osher and E. Fatemi, Nonlinear Total Variation based Noise Removal Algorithms, Physica D. 60 (1992), 259-268. 
[44] L. Silvestre. Hölder estimates for solutions of integro differential equations like the fractional laplace. Indiana Univ. Math. J. 55 (2006), 1155-1174.

[45] L. Silvestre. Regularity of the obstacle problem for a fractional power of the Laplace operator. Comm. Pure Appl. Math. 60 (2007), 67-112.

[46] D. Strong and T. Chan, Relation of regularization parameter and scale in total variation based image denoising, UCLACAM Report 96-7, University of California, Los Angeles, CA, 1996.

[47] J. M. Urbano, The method of intrinsic scaling. A systematic approach to regularity for degenerate and singular PDEs. Lecture Notes in Mathematics, 1930. Springer-Verlag, Berlin, 2008.

[48] J. L. Vázquez, Smoothing and decay estimates for nonlinear diffusion equations. Equations of porous medium type. Oxford Lecture Series in Mathematics and its Applications, 33. Oxford University Press, Oxford, 2006.

[49] X. Wang. Metaestability and stability of patterns in a convolution model for phase transitions. J. Differential Equations 183 (2002), 434-461.

[50] L. Zhang. Existence, uniqueness and exponential stability of traveling wave solutions of some integral differential equations arising from neuronal networks. J. Differential Equations, 197 (2004), 162-196.

[51] W. P. Ziemer, Weakly Differentiable Functions, GTM 120, Springer Verlag, 1989.

Fuensanta Andreu, José M. Mazón and J. Julián Toledo-Melero

DePARTAMEnt D'AnÀlisi Matemàtica, Universitat de VAlÈnCia

VALENCIA, SPAIN.

E-mail address: fuensanta.andreu@uv.es, mazon@uv.es, toledojj@uv.es

Web page: http://www.uv.es/ mazon

Julio D. Rossi

Departamento de Matemática, FCEyn UBA (1428)

Buenos Aires, Argentina.

E-mail address: jrossi@dm.uba.ar

Web page: http://mate.dm.uba.ar/ jrossi 